\title{
Controllability of analytic functions for a wave equation coupled with a beam
}

Brice Allibert and Sorin Micu

Abstract. We consider the controllability and observation problem for a simple model describing the interaction between a fluid and a beam. For this model, microlocal propagation of singularities proves that the space of controlled functions is smaller that the energy space. We use spectral properties and an explicit construction of biorthogonal sequences to show that analytic functions can be controlled within finite time. We also give an estimate for this time, related to the amount of analyticity of the latter function.

\section{Introduction.}

Let $\Omega$ be the two-dimensional square $\Omega=(0,1) \times(0,1) \subset \mathbb{R}^{2}$.

We assume that $\Omega$ is filled with an elastic, inviscid, compressible fluid whose velocity field $\vec{v}$ is given by the potential $\Phi=\Phi(x, y, t)$, $\vec{v}=\nabla \Phi$. By linearization we assume that the potential $\Phi$ satisfies the linear wave equation in $\Omega \times(0, \infty)$.

The boundary $\Gamma=\partial \Omega$ of $\Omega$ is divided in two parts $\Gamma_{0}=\{(0, y)$ : $y \in(0,1)\}$ and $\Gamma_{1}=\Gamma \backslash \Gamma_{0}$. The subset $\Gamma_{1}$ is assumed to be rigid and we impose zero normal velocity of the fluid on it. The subset $\Gamma_{0}$ is supposed to be flexible and occupied by a Bernoulli-Euler beam that 
vibrates under the pressure of the fluid on the plane where $\Omega$ lies. The displacement of $\Gamma_{0}$ is described by the scalar function $W=W(y, t)$. On the other hand, on $\Gamma_{0}$ we impose the continuity of the normal velocities of the fluid and the beam. The beam is assumed to satisfy Neumann-type boundary conditions on its extremes. All deformations are supposed to be small enough so that linear theory applies. Under natural initial conditions for $\Phi$ and $W$ the linear motion of this system is described by means of the following coupled equations

$$
\begin{cases}\Phi_{t t}-\Delta \Phi=0, & \text { in } \Omega \times(0, \infty), \\ \frac{\partial \Phi}{\partial \nu}=0, & \text { on } \Gamma_{1} \times(0, \infty), \\ \frac{\partial \Phi}{\partial x}=-W_{t}, & \text { on } \Gamma_{0} \times(0, \infty), \\ W_{t t}+W_{y y y y}+\Phi_{t}=0, & \text { on } \Gamma_{0} \times(0, \infty), \\ W_{y}(0, t)=W_{y}(1, t)=0, & \text { for } t>0, \\ W_{y y y}(0, t)=W_{y y y}(1, t)=0, & \text { for } t>0, \\ \Phi(0)=\Phi^{0}, \Phi_{t}(0)=\Phi^{1}, & \text { in } \Omega, \\ W(0)=W^{0}, W_{t}(0)=W^{1}, & \text { on } \Gamma_{0} .\end{cases}
$$

By $\nu$ we denote the unit outward normal to $\Omega$.

In (1) we have chosen to take the various parameters of the system to be equal to one.

System (1) is well-posed in the energy space $\mathcal{Y}=H^{1}(\Omega) \times L^{2}(\Omega) \times$ $H_{N}^{2}\left(\Gamma_{0}\right) \times L^{2}\left(\Gamma_{0}\right)$ for the variables $\left(\Phi, \Phi_{t}, W, W_{t}\right)$ where $H_{N}^{2}\left(\Gamma_{0}\right)=\{v \in$ $\left.H^{2}(0,1): v_{y}(0)=v_{y}(1)=0\right\}$. The energy

$$
E(t)=\frac{1}{2} \int_{\Omega}\left(|\nabla \Phi|^{2}+\left|\Phi_{t}\right|^{2}\right) d x d y+\frac{1}{2} \int_{\Gamma_{0}}\left(\left|W_{y y}\right|^{2}+\left|W_{t}\right|^{2}\right) d y
$$

remains constant along trajectories.

It is easy to see that the equilibria of these systems are of the form

$$
\left(\Phi, \Phi_{t}, W, W_{t}\right)=\left(c_{1}, 0, c_{2}, 0\right)
$$

$c_{1}$ and $c_{2}$ being constant functions.

We study the controllability of system (1) under the action of an exterior force on the flexible part of the boundary $\Gamma_{0}$. The control is 
given by a scalar function $\beta=\beta(y, t)$ in the space $H^{-2}\left(0, T ; L^{2}\left(\Gamma_{0}\right)\right)$. Of course this is an arbitrary choice and many others make sense. However this is the most natural one when solving the control problem by means of J. L. Lions's HUM (see [6]), as we will do. The controlled system reads as follows

$$
\begin{cases}\Phi_{t t}-\Delta \Phi=0, & \text { in } \Omega \times(0, \infty), \\ \frac{\partial \Phi}{\partial \nu}=0, & \text { on } \Gamma_{1} \times(0, \infty), \\ \frac{\partial \Phi}{\partial x}=-W_{t}, & \text { on } \Gamma_{0} \times(0, \infty), \\ W_{t t}+W_{y y y}+\Phi_{t}=\beta, & \text { on } \Gamma_{0} \times(0, \infty), \\ W_{y}(0, t)=W_{y}(1, t)=0, & \text { for } t>0, \\ W_{y y y}(0, t)=W_{y y y}(1, t)=0, & \text { for } t>0, \\ \Phi(0)=\Phi^{0}, \Phi_{t}(0)=\Phi^{1}, & \text { in } \Omega, \\ W(0)=W^{0}, W_{t}(0)=W^{1}, & \text { on } \Gamma_{0} .\end{cases}
$$

The problem of controllability can be formulated as follows: Given $T>2$, find the space of initial data $\left(\Phi^{0}, \Phi^{1}, W^{0}, W^{1}\right)$ that can be driven to an equilibrium of the form (3) in time $T$ by means of a suitable control $\beta \in H^{-2}\left(0, T ; L^{2}\left(\Gamma_{0}\right)\right)$.

The model under consideration is inspired in and related to that of H. T. Banks et al. in [4]. However, there are some important differences between these two models. First of all, we choose Neumann-type boundary conditions for the beam. These are compatible with those of $\Phi$ in order to develop solutions in Fourier series. Another difference is related to the nature of the controls. In [4] the control acts on the system through a finite number of piezoceramic patches located on $\Gamma_{0}$. This restricts very much the set of admissible controls, that are essentially second derivatives of Heaviside functions, and much weaker controllability results have to be expected. In [4] the controllability problem is not addressed. Instead, they consider a quadratic optimal control problem. More recently in [2] a Riccati equation for the optimal control is derived. The problem of the controllability of one-dimensional beams with piezoelectric actuators has been successfully addressed by M. Tucsnak [9]. However, to our knowledge, there are no rigorous results on the controllability of fluid-structure systems under such controls. In [7] the controllability problem for a similar system with a string instead 
of a beam was studied. It was shown that a space of analytical initial data can be controlled in any time $T>2$. The techniques we develop in the present article can be applied to that case and allow to show that larger and larger classes of analytic functions can be controlled in finite time.

The propagation of singularities for the wave equation on any segment parallel to $\Gamma_{0}$ proves that the space of controlled functions will be small. It will not contain all functions of finite energy.

Let us denote by $\mathcal{X}=H^{1}(0,1) \times L^{2}(0,1) \times \mathbb{C} \times \mathbb{C}$ and by $\mathcal{X}^{\prime}$ its dual space. Let also $\mathcal{Y}^{n}=\left(H^{1}(0,1) \times L^{2}(0,1) \times \mathbb{C} \times \mathbb{C}\right) \cos (n \pi y)$.

By the HUM method, we will first prove that if $C(n, T)$ is a sequence of constants such that any solution of the observation problem

$$
\begin{cases}\Phi_{t t}-\Delta \Phi=0, & \text { in } \Omega \times(0, \infty), \\ \frac{\partial \Phi}{\partial \nu}=0, & \text { on } \Gamma_{1} \times(0, \infty), \\ \frac{\partial \Phi}{\partial x}=W_{t}, & \text { on } \Gamma_{0} \times(0, \infty), \\ W_{t t}+W_{y y y}-\Phi_{t}=0, & \text { on } \Gamma_{0} \times(0, \infty), \\ W_{y}(0, t)=W_{y}(1, t)=0, & \text { for } t>0, \\ W_{y y y}(0, t)=W_{y y y}(1, t)=0, & \text { for } t>0, \\ \left.\left(\Phi, \Phi_{t}\right)\right|_{t=0}=\left(\Phi^{0}, \Phi^{1}\right), & \text { in } \Omega, \\ \left.\left(W, W_{t}\right)\right|_{t=0}=\left(W^{0}, W^{1}\right), & \text { on } \Gamma_{0},\end{cases}
$$

with initial conditions in $\mathcal{Y}^{n}$, satisfies

$$
\left\|\left(\Phi^{0}, \Phi^{1}, W^{0}, W^{1}\right)\right\|_{\mathcal{Y}}^{2} \leq C(n, T) \int_{-T}^{T}\left|W_{t t}(0, t)\right|^{2} d t
$$

then the space of initial data

$$
\begin{aligned}
H=\{ & \sum_{n}\left(\Phi^{0}, \Phi^{1}, W^{0}, W^{1}\right)_{n} \cos (n \pi y) \mid\left(\Phi^{0}, \Phi^{1}, W^{0}, W^{1}\right)_{n} \in \mathcal{X} \\
& \text { such that } \left.\sum_{n} C(n, T)\left(\left\|\left(\Phi^{0}, \Phi^{1}, W^{0}, W^{1}\right)_{n}\right\|_{\mathcal{X}^{\prime}}^{2}+\left|\Phi^{0}(0)\right|^{2}\right)<\infty\right\}
\end{aligned}
$$

is a subset of the space of controlled functions. Remark that the space $H$ depends on the constants $C(n, T)$ : when $C(n, T)$ "increase", $H$ becomes smaller. 
This paper aims at proving that, for $T$ and $n$ large enough,

$$
C(n, T) \leq C e^{\alpha(T)|n|}
$$

with the following property

Theorem 1. For any positive real number $q$, there is a constant $C_{q}$ such that

$$
\alpha(T) \leq \frac{C_{q}}{T^{1-q}}
$$

It means that any initial condition whose Fourier coefficients in $y$ decrease like $e^{-|n| \alpha}$ can be controlled if $T$ is larger than $T(\alpha)=$ $\sqrt[1-q]{C_{q} / \alpha}$. This condition on the Fourier coefficients means that the initial condition is analytic with respect to $y$ and that it can be continued as an holomorphic function over the complex strip $|\operatorname{Im} y|<\alpha$.

Now any initial condition that is analytic with respect to $y$ can be continued as an holomorphic function over a such a strip $|\operatorname{Im} y|<\varepsilon$ for a positive $\varepsilon$ that depends on this initial condition. Therefore, its Fourier coefficients with respect to $y$ decrease like $e^{-|n| \varepsilon}$. So according to Theorem 1 and (6), it can be controlled if $T>T(\varepsilon)$.

This means that any initial condition of finite energy that is analytic with respect to $y$ can be controlled in a finite time (which is not uniform).

It is important to notice that analyticity is required only with respect to the variable $y$. Therefore the space of controlled functions is not symmetric in $x$ and $y$. This means that we do not use the fact that the metrics in our problem is analytic with respect to $x$. In [1], the boundary control problem is studied on a surface of revolution. The same kind of result is proved in that case, even if the surfece is only $\mathcal{C}^{\infty}$. This is posible because such surface is still "analytic" with respect to the angular variable, even if it is only $\mathcal{C}^{\infty}$ with respect to its axial variable.

The rest of the article is organized as follows. In Section 2 we give a direct estimate for the observation problem and, by using (6), we apply Hilbert Uniqueness Method to solve our controllability problem. We obtain that the initial data from $H$ can be controlled in time $T$. In Section 3 we prove some spectral properties of the operator that will be used in the proof of the main theorem in Section 4. In the last section an explicit dependence of the space $H$ on the time $T$ is obtained. 


\section{The direct estimate and the controllability problem.}

\subsection{Direct estimate.}

Let us consider the system

$$
\begin{cases}\eta_{t t}-\eta_{x x}+n^{2} \pi^{2} \eta=f, & \text { in }(0,1) \times(0, T), \\ \eta_{x}(1)=0, & \text { for } t \in(0, T), \\ \eta_{x}(0)=u_{t}, & \text { for } t \in(0, T), \\ u_{t t}+n^{4} \pi^{4} u-\eta_{t}(0)=g, & \text { for } t \in(0, T), \\ \eta(0)=\eta^{0}, \eta_{t}(0)=\eta^{1}, & \text { in }(0,1), \\ u(0)=u^{0}, u_{t}(0)=u^{1} . & \end{cases}
$$

The unknowns are $\eta=\eta(x, t)$ and $u=u(t)$. Of course, since the coefficients of the system depend on $n=0,1, \ldots$, solutions $(\eta, u)$ depend on $n$ too. However, in order to simplify the notations we will not use the index $n$ to distinguish the solutions of (8) for the different values of $n$.

The energy space for system $(8)$ is the Hilbert space $\mathcal{X}=H^{1}(0,1) \times$ $L^{2}(0,1) \times \mathbb{C} \times \mathbb{C}$.

It is easy to see that for any $\left(\eta^{0}, \eta^{1}, u^{0}, u^{1}\right) \in \mathcal{X}$ and $(f, g) \in$ $L^{1}\left(0, T ; L^{2}(0,1) \times \mathbb{C}\right)$ system $(8)$ has a unique solution in the class

(9) $\eta \in C\left([0, T] ; H^{1}(0,1)\right) \cap C^{1}\left([0, T] ; L^{2}(0,1)\right) ; u \in C^{1}([0, T] ; \mathbb{C})$.

In other words $\left(\eta, \eta_{t}, u, u_{t}\right) \in C([0, T] ; \mathcal{X})$.

The energy of the system

$$
F(t)=\frac{1}{2} \int_{0}^{1}\left(\left|\eta_{t}\right|^{2}+\left|\eta_{x}\right|^{2}+n^{2} \pi^{2} \eta^{2}\right) d x+\frac{1}{2}\left(\left|u_{t}\right|^{2}+n^{4} \pi^{4}|u|^{2}\right)
$$

satisfies

$$
\frac{d F(t)}{d t}=\int_{0}^{1} f(x, t) \eta_{t}(x, t) d x+g(t) u_{t}(t) .
$$

Therefore, when $f \equiv 0$ and $g \equiv 0$, the energy $F$ remains constant along trajectories.

We observe that when $n \geq 1$ the square root of $F$ defines a norm in $\mathcal{X}$ equivalent to the canonical norm $\|\cdot\|_{\mathcal{X}}$ of $\mathcal{X}$

$$
\|(u, v, w, z,)\|_{\mathcal{X}}=\left(\int_{0}^{1}\left(\left|u_{x}\right|^{2}+|u|^{2}+|v|^{2}\right) d x+w^{2}+z^{2}\right)^{1 / 2}
$$


However, when $n=0$ this is not the case. Actually, for $n=0,(\eta, u)=$ $\left(c_{1}, c_{2}\right)$ with $c_{1}, c_{2}$ real constants are stationary solutions of (8) with $f \equiv 0, g \equiv 0$ for which the energy $F$ vanishes.

We have the following "hidden regularity" result

Proposition 1. For any $T>0$ there exists a constant $C(T)>0$ independent of $n=0,1, \ldots$ such that

$$
\begin{gathered}
\left(\int_{0}^{T}\left|u_{t t}\right| d t\right)^{2}+\int_{0}^{T}\left(\left|u_{t}\right|^{2}+\left(1+n^{8} \pi^{8}\right) u^{2}+\left(1+n^{2} \pi^{2}\right) \eta^{2}(0, t)\right) d t \\
\leq C\left(n^{4}+1\right)\left(\left\|\left(\eta^{0}, \eta^{1}, u^{0}, u^{1}\right)\right\|_{\mathcal{X}}^{2}\right. \\
\left.+\|f\|_{L^{1}\left(0, T ; L^{2}(0,1)\right)}^{2}+\|g\|_{L^{1}(0, T)}^{2}\right),
\end{gathered}
$$

for any $\left(\eta^{0}, \eta^{1}, u^{0}, u^{1}\right) \in \mathcal{X}, f \in L^{1}\left(0, T ; L^{2}(0,1)\right)$ and $g \in L^{1}(0, T)$.

If $g \in L^{2}(0, T)$, then $u \in H^{2}(0, T)$ and we also have

$$
\begin{aligned}
& \int_{0}^{T}\left|u_{t t}\right|^{2} d t \leq C\left(n^{4}+1\right)\left(\left\|\left(\eta^{0}, \eta^{1}, u^{0}, u^{1}\right)\right\|_{\mathcal{X}}^{2}\right. \\
&\left.+\|f\|_{L^{1}\left(0, T ; L^{2}(0,1)\right)}^{2}+\|g\|_{L^{2}(0, T)}^{2}\right) .
\end{aligned}
$$

REMARK. This proposition shows that $u$ is more smooth than what (9) guarantees. This is due to the structure of the second order (in time) equation that $u$ satisfies. The fact that the constant $c$ in (13) and (14) does not depend on the index $n$ is worth mentioning.

Proof of Proposition 1. It is enough to consider smooth solutions since a classical density argument allows to extend inequalities (13) and (14) to any solution with finite right hand side. We use a classical multiplier technique (see, for instance, [6]). We multiply the first equation in $(8)$ by $(1-x) \eta_{x}$ and integrate over $(0,1) \times(0, T)$. Integrating by parts we obtain

$$
\begin{aligned}
& \frac{1}{2} \int_{0}^{T}\left(\left|\eta_{t}\right|^{2}+\left|\eta_{x}\right|^{2}-n^{2} \pi^{2} \eta^{2}\right)(0, t) d t \\
& =-\left.\int_{0}^{1} \eta_{t}(1-x) \eta_{x} d x\right|_{0} ^{T} \\
& \quad+\frac{1}{2} \int_{0}^{T} \int_{0}^{1}\left(\eta_{t}^{2}+\eta_{x}^{2}-n^{2} \pi^{2} \eta^{2}\right) d x d t+\int_{0}^{T} \int_{0}^{1} f(1-x) \eta_{x} d x d t \\
& =X .
\end{aligned}
$$


In this identity we use the notation $\left.L\right|_{0} ^{T}=L(T)-L(0)$. The right hand side of this identity can be easily bounded as follows

$$
\begin{aligned}
|X| \leq & \frac{1}{2} \int_{0}^{1}\left(\eta_{t}^{2}+\eta_{x}^{2}\right)(x, 0) d x+\frac{1}{2} \int_{0}^{1}\left(\eta_{t}^{1}+\eta_{x}^{2}\right)(x, T) d x \\
& +\int_{0}^{T} F(t) d t+\frac{1}{2}\left(\|f\|_{L^{1}\left(0, T ; L^{2}(0,1)\right)}^{2}+\left\|\eta_{x}\right\|_{L^{\infty}\left(0, T ; L^{2}(0,1)\right)}^{2}\right) \\
\leq & F(0)+F(T)+\int_{0}^{T} F(t) d t+\|F(t)\|_{L^{\infty}(0, T)}+\frac{1}{2}\|f\|_{L^{1}\left(0, T ; L^{2}(0,1)\right)}^{2} \\
\leq & C\left(\|F\|_{L^{\infty}(0, T)}+\|f\|_{L^{1}\left(0, T ; L^{2}(0,1)\right)}^{2}\right)
\end{aligned}
$$

with $C>0$ independent of $n$.

In the sequel, if some constant in the inequalities depends on $n$, we will make it explicit by means of an index $n$ on that constant.

On the other hand, from identity (11) and using Gronwall's inequality it is easy to deduce that

$$
\|F\|_{L^{\infty}(0, T)}^{2} \leq C\left(\|f\|_{L^{1}\left(0, T ; L^{2}(0,1)\right)}^{2}+\|g\|_{L^{1}(0, T)}^{2}+F(0)\right) .
$$

Since $H^{1}(0,1)$ is continuously embedded in $C([0,1] ; \mathbb{C})$ we also have

$$
\begin{aligned}
\int_{0}^{T} \eta^{2}(0, t) d t & \leq C \int_{0}^{T} F(t) d t \\
& \leq C\left(\|f\|_{L^{1}\left(0, T ; L^{2}(0,1)\right)}^{2}+\|g\|_{L^{1}(0, T)}^{2}+F(0)\right) .
\end{aligned}
$$

Combining these inequalities we deduce that

$$
\begin{aligned}
\int_{0}^{T}\left(\left|\eta_{t}\right|^{2}\right. & \left.+\left|\eta_{x}\right|^{2}+n^{2} \pi^{2} \eta^{2}\right)(0, t) d t \\
\leq C\left(n^{2}+1\right) & \left(\left\|\left(\eta^{0}, \eta^{1}, u^{0}, u^{1}\right)\right\|_{\mathcal{X}}^{2}\right. \\
& \left.+\|f\|_{L^{1}\left(0, T ; L^{2}(0,1)\right)}^{2}+\|g\|_{L^{1}(0, T)}^{2}\right) .
\end{aligned}
$$

On the other hand

$$
\begin{aligned}
n^{8} \pi^{8} \int_{0}^{T} u^{2}(t) d t & \leq 2 n^{4} \pi^{4} \int_{0}^{T} F(t) d t \\
\leq C n^{4}( & \left\|\left(\eta^{0}, \eta^{1}, u^{0}, u^{1}\right)\right\|_{\mathcal{X}}^{2} \\
& \left.\quad+\|f\|_{L^{1}\left(0, T ; L^{2}(0,1)\right)}^{2}+\|g\|_{L^{1}(0, T)}^{2}\right) .
\end{aligned}
$$


Inequalities (13) and (14) are a direct consequence of (15) and (16) and the coupling conditions between $\eta$ and $u$ given in system (8), i.e.

$$
\eta_{y}(0, t)=u_{t}(t), \quad u_{t t}(t)=g(t)+\eta_{t}(0, t)-n^{4} \pi^{4} u(t),
$$

for $t \in(0, T)$.

\subsection{A controllability result.}

In this section, we solve the controllability problem (4) stated in the Introduction by using J.- L. Lions's HUM. This is done by Fourier descomposition which is possible because of the boundary conditions we have chosen for $W$. Indeed, $W$ is assumed to satisfy Neumann type boundary conditions which are compatible with those of $\Phi$ to develop solutions in Fourier series.

Indeed, let us decompose the control $\beta$, the solutions $\Phi, W$ and the initial data in the following way

$$
\left\{\begin{array}{l}
\beta=\sum_{n=0}^{\infty} \beta_{n}(t) \cos (n \pi y) \\
\Phi=\sum_{n=0}^{\infty} \Psi_{n}(x, t) \cos (n \pi y) \\
\left(\Phi^{0}, \Phi^{1}\right)=\sum_{n=0}^{\infty}\left(\Psi_{n}^{0}(x), \Psi_{n}^{1}(x)\right) \cos (n \pi y), \\
W=\sum_{n=0}^{\infty} V_{n}(t) \cos (n \pi y) \\
\left(W^{0}, W^{1}\right)=\sum_{n=0}^{\infty}\left(V_{n}^{0}, V_{n}^{1}\right) \cos (n \pi y) .
\end{array}\right.
$$

With this decomposition, system (4) can be split into the following sequence of one-dimensional controlled systems for $n=0,1, \ldots$

$$
\begin{array}{ll}
\Psi_{n, t t}-\Psi_{n, x x}+n^{2} \pi^{2} \Psi_{n}=0, & \text { in }(0,1) \times(0, \infty), \\
\Psi_{n, x}(1, t)=0, & \text { for } t>0, \\
\Psi_{n, x}(0, t)=-V_{t}(t), & \text { for } t>0,
\end{array}
$$




$$
\begin{array}{ll}
V_{n, t t}(t)+n^{4} \pi^{4} V_{n}(t) & \\
+\Psi_{n, t}(0, t)=\beta_{n}(t), & \text { for } t>0, \\
\Psi_{n}(0)=\Psi_{n}^{0}, \Psi_{n, t}(0)=\Psi_{n}^{1}, & \text { in }(0,1), \\
V_{n}(0)=V_{n}^{0}, V_{n, t}(0)=V_{n}^{1} . &
\end{array}
$$

The control $\beta$ we obtain is of the form

$$
\beta=\frac{\partial^{2}}{\partial t^{2}} \gamma
$$

with $\gamma \in L^{2}\left(\Gamma_{0} \times(0, T)\right)$ having compact support in time. Therefore $\int_{0}^{T} \beta=0$. Taking this fact into account it is easy to see that the constants $c_{1}, c_{2}$ of the equilibrium we reach at time $t=T$ are determined a priori by the initial data. Indeed, integrating the first equation of (4) in $\Omega$ we obtain that

$$
\int_{\Omega} \Phi_{t} d x d x-\int_{\Gamma_{0}} W d y
$$

remains constant in time. Therefore, necessarily,

$$
c_{2}=\int_{\Gamma_{0}} W^{0} d y-\int_{\Omega} \Phi^{1} d x d y
$$

On the other hand, integrating the equation satisfied by $W$ on $\Gamma_{0} \times(0, T)$ and taking into account that $\int_{0}^{T} \beta=0$ we deduce that

$$
\int_{\Gamma_{0}} W_{t}(T) d y+\int_{\Gamma_{0}} \Phi(0, y, T) d y=\int_{\Gamma_{0}} W^{1} d y+\int_{\Gamma_{0}} \Phi^{0}(y, 0) d y
$$

and therefore

$$
c_{1}=\int_{\Gamma_{0}}\left(W^{1}+\Phi^{0}(0, y)\right) d y .
$$

In terms of the Fourier coefficients (18) these constants can be written in the following way

$$
c_{1}=V_{0}^{1}+\Psi_{0}^{0}(0), \quad c_{2}=V_{0}^{0}-\int_{0}^{1} \Psi_{0}^{1}(x) d x .
$$

Therefore, the constants $c_{1}$ and $c_{2}$ of the equilibrium we may reach are uniquely determined by the Fourier coefficients of the initial data corresponding to the frequency $n=0$ in the $y$-variable. 
This fact is related to the different nature of systems (19) for $n=0$ and $n \geq 1$. While for any $n \geq 1$ system (19) is exactly controllable to zero at any time $T>2$, when $n=0$ we can only control the system to the equilibrium given by (22) in terms of the initial data.

In this section we suppose that for any $n \in \mathbb{N}^{*}$ and time $T>2$ we can find a constant $C(n, T)$ such that for any $\left(\Psi^{0}, \Psi^{1}, V^{0}, V^{1}\right) \in \mathcal{X}$, the solution of problem

$$
\begin{cases}\Psi_{t t}-\Psi_{x x}+n^{2} \pi^{2} \Psi=0, & \text { in }(0,1) \times(0, \infty) \\ \Psi_{x}(1, t)=0, & \text { for } t>0 \\ \Psi_{x}(0, t)=V_{t}(t), & \text { for } t>0 \\ V_{t t}(t)+n^{4} \pi^{4} V(t)-\Psi_{t}(0, t)=0, & \text { for } t>0 \\ \Psi(0)=\Psi^{0}, \Psi_{t}(0)=\Psi^{1}, & \text { in }(0,1) \\ V(0)=V^{0}, V_{t}(0)=V^{1} & \end{cases}
$$

satisfies

$$
\left\|\left(\Psi^{0}, \Psi^{1}, V^{0}, V^{1}\right)\right\|_{\mathcal{X}}^{2} \leq C(n, T) \int_{0}^{T}\left|V_{t t}\right|^{2} d t .
$$

We shall prove (24) and we shall give estimates over $C(n, T)$ in Section 4, while proving Theorem 1 .

When $n \geq 1$ we have the following controllability result for (19).

Proposition 2. Let $\mathcal{X}$ be the space $H^{1}(0,1) \times L^{2}(0,1) \times \mathbb{C} \times \mathbb{C}$. Assume that $T>2$ and $n \geq 1$. Then, for any $\left(\Psi^{1}, \Psi^{0}, V^{1}, V^{0}\right) \in \mathcal{X}$, there exists a control $\beta \in H^{-2}(0, T)$ with compact support such that the solution $(\Psi, V)$ of (19) satisfies

$$
\Psi(T)=\Psi_{t}(T) \equiv 0 \text { in }(0,1), \quad V(T)=V_{t}(T)=0 .
$$

REMARK. In the statement of Proposition 2 and in the sequel we drop the index $n$ from the unknowns $(\Psi, V)$ to simplify the notation.

The solution $(\Psi, V)$ is defined by transposition. Therefore (25) has to be understood in a suitable weak sense. We will return to this question in the proof of the proposition.

The proof of Proposition 2 provides the continuous dependence of the control $\beta$ on the initial data. More precisely

$$
\|\beta\|_{H^{-2}(0, T)}^{2} \leq C(n, T)\left(\left\|\left(\Psi^{1}, \Psi^{0}, V^{1}, V^{0}\right)\right\|_{\mathcal{X}^{\prime}}^{2}+\left|\Psi^{0}(0)\right|^{2}\right),
$$


for any initial data $\left(\Psi^{0}, \Psi^{1}, V^{0}, V^{1}\right)$ as in the statement of Proposition 2. By $\mathcal{X}^{\prime}$ we denote the dual of the space $\mathcal{X}$. The constant $C(n, T)$ in (26) is the one appearing in (24) and will be evaluated in Section 4.

Proof. We use HUM to prove this result.

Given any $\left(\eta^{0}, \eta^{1}, u^{0}, u^{1}\right) \in \mathcal{X}$ we solve the adjoint system

$$
\begin{cases}\eta_{t t}-\eta_{x x}+n^{2} \pi^{2} \eta=0, & \text { in }(0,1) \times(0, T), \\ \eta_{x}(1, t)=0, & \text { for } t \in(0, T), \\ \eta_{x}(0, t)=u_{t}(t), & \text { for } t \in(0, T) \\ u_{t t}(t)+n^{4} \pi^{4} u(t)-\eta_{t}(0, t)=0, & \text { for } t \in(0, T) \\ \eta(0)=\eta^{0}, \eta_{t}(0)=\eta^{1}, & \text { in }(0,1) \\ u(0)=u^{0}, u_{t}(0)=u^{1} & \end{cases}
$$

We fix, some non-negative smooth function $\rho(0, T) \longrightarrow \mathbb{R}$ with compact support such that $\rho \equiv 1$ in $(\varepsilon, T-\varepsilon)$ with $T-2 \varepsilon>2$.

We then solve the backward system

$$
\begin{cases}\Psi_{t t}-\Psi_{x x}+n^{2} \pi^{2} \Psi=0, & \text { in }(0,1) \times(0, T), \\ \Psi_{x}(1, t)=0, & \text { for } t \in(0, T), \\ \Psi_{x}(0, t)=-V_{t}(t), & \text { for } t \in(0, T), \\ V_{t t}+n^{4} \pi^{4} V+\Psi_{t}(0, t) & \\ =-\frac{d^{2}}{d t^{2}}\left(\rho(t) u_{t t}(t)\right), & \text { for } t \in(0, T), \\ \Psi(T)=\Psi_{t}(T)=0, & \text { in }(0,1), \\ V(T)=V_{t}(T)=0 . & \end{cases}
$$

The solution of (28) is defined by transposition (see [6]). If we multiply in (28) by any solution $(\widetilde{\eta}, \widetilde{u})$ of (8) and integrate (formally) by parts we obtain the following identity

$$
\begin{gathered}
\int_{0}^{T} \rho(t) u_{t t}(t) \widetilde{u}_{t t}(t) d t+\int_{0}^{T} \int_{0}^{1} \tilde{f} \Psi d x d t-\int_{0}^{T} \widetilde{g} V d t \\
=\int_{0}^{1}\left(-\Psi_{t}(0) \widetilde{\eta}(0)+\Psi(0) \widetilde{\eta}_{t}(0)\right) d x+V(0) \widetilde{\eta}(0,0) \\
+\Psi(0,0) \widetilde{u}(0)-V(0) \widetilde{u}_{t}(0)+V_{t}(0) \widetilde{u}(0)
\end{gathered}
$$


Notice that when we derived (29) we have used the fact that $\rho$ and its first derivative vanish for $t=0$ and $T$.

We adopt (29) as definition of weak solution in the sense of transposition of (28). More precisely we say that $(\Psi, V)$ solve (28) if (29) holds for any $\left(\widetilde{\eta}^{0}, \widetilde{\eta}^{1}, \widetilde{u}^{0}, \widetilde{u}^{1}\right) \in \mathcal{X}$ and $(\widetilde{f}, \widetilde{g}) \in L^{1}\left(0, T ; L^{2}(0,1) \times \mathbb{C}\right)$.

We observe that $(29)$ can be rewritten in the following way

$$
\begin{aligned}
\int_{0}^{T} \rho(t) u_{t t}(t) \widetilde{u}_{t t} d t-\int_{0}^{T} \int_{0}^{1} \widetilde{f} \Psi d x d t+\int_{0}^{T} \widetilde{g} V d t \\
=-\left\langle\Psi_{t}(0)+V(0) \delta_{0}, \widetilde{\eta}(0)\right\rangle+\left\langle\Psi(0), \widetilde{\eta}_{t}(0)\right\rangle \\
+\left(V_{t}(0)+\Psi(0,0)\right) \widetilde{u}(0)-V(0) \widetilde{u}_{t}(0)
\end{aligned}
$$

where $\langle\cdot, \cdot\rangle$ denotes both the duality pairing between $\left(H^{1}(0,1)\right)^{\prime}$ and $H^{1}(0,1)$ and the scalar product in $L^{2}(0,1)$ and $\delta_{0} \in\left(H^{1}(0,1)\right)^{\prime}$ denotes the Dirac delta at $y=0$.

We have the following existence and uniqueness result of solutions in the sense of transposition.

Proposition 3. System (28) has a unique solution in the sense of transposition. More precisely, for any solution $(\eta, u)$ of $(27)$ with initial data in $\mathcal{X}$, there exists a unique $(\Psi, V) \in C\left([0, T] ; L^{2}(0,1)\right) \times L^{2}(0, T)$, $\rho^{0} \in L^{2}(0,1), \rho^{1} \in\left(H^{1}(0,1)\right)^{\prime}, \mu^{0} \in \mathbb{C}, \mu^{1} \in \mathbb{C}$ satisfying

$$
\begin{aligned}
\int_{0}^{T} \rho(t) u_{t t}(t) \widetilde{u}_{t t} d t= & \int_{0}^{T} \int_{0}^{1} \tilde{f} \Psi d x d t-\int_{0}^{T} \widetilde{g} V d t \\
& +\left\langle\rho^{1}, \widetilde{\eta}(0)\right\rangle+\left\langle\rho^{0}, \widetilde{\eta}_{t}(0)\right\rangle+\mu^{1} \widetilde{u}(0)+\mu^{0} \widetilde{u}_{t}(0)
\end{aligned}
$$

for any solution $(\widetilde{\eta}, \widetilde{u})$ of $(8)$ with

$$
\left(\widetilde{\eta}^{0}, \widetilde{\eta}^{1}, \widetilde{u}^{0}, \widetilde{u}^{1}\right) \in \mathcal{X}, \tilde{f} \in L^{1}\left(0, T ; L^{2}(0,1)\right), \widetilde{g} \in L^{2}(0,1) .
$$

REMARK. In the identity (31) $\rho^{0}, \rho^{1}, \mu^{0}$ and $\mu^{1}$ play respectively the role of $\Psi(0),-\Psi_{t}(0)+V(0) \delta_{0},-V(0)$ and $V_{t}(0)+\Psi(0,0)$. It is easy to see that, in the frame of smooth functions, there is a one to one correspondence between $\left(\rho^{0}, \rho^{1}, \mu^{0}, \mu^{1}\right)$ and $\left(\Psi(0), \Psi_{t}(0), V(0), V_{t}(0)\right)$.

Proof of Proposition 3. In view of Proposition 1 we know that the map

$$
\left(\widetilde{\eta}^{0}, \widetilde{\eta}^{1}, \widetilde{u}^{0}, \widetilde{u}^{1}, \widetilde{f}, \widetilde{g}\right) \longrightarrow \int_{0}^{T} \rho(t) u_{t t}(t) \widetilde{u}_{t t}(t) d t
$$


is linear and continuous from $\mathcal{X} \times L^{1}\left(0, T ; L^{2}(0,1)\right) \times L^{2}(0, T)$ into $\mathbb{C}$. This implies the existence and uniqueness of $\left(\rho^{1}, \rho^{0}, \mu^{1}, \mu^{0}\right) \times(\Psi, V) \in$ $\mathcal{X}^{\prime} \times L^{\infty}\left(0, T ; L^{2}(0,1)\right) \times L^{2}(0, T)$ such that $(31)$ holds. Moreover, there exists a constant $C>0$ such that

$$
\begin{aligned}
\|(\Psi, V)\|_{L^{\infty}\left(0, T ; L^{2}(0,1)\right) \times L^{2}(0, T)}+ & \left\|\left(\rho^{1}, \rho^{0}, \mu^{1}, \mu^{0}\right)\right\|_{\mathcal{X}^{\prime}} \\
& \leq C\left\|u_{t t}\right\|_{L^{2}(0, T)} \\
& \leq C\left\|\left(\eta^{0}, \eta^{1}, u^{0}, u^{1}\right)\right\|_{\mathcal{X}^{\prime}} .
\end{aligned}
$$

The fact that $\Psi \in C\left([0, T] ; L^{2}(0,1)\right)$ can be deduced from (33) by a classical density argument.

REMARK 4. When the data of (27) are smooth, the solution $(\eta, u)$ is smooth too. It is easy to see that (28) has a finite energy solution. In this case one can check that the element $\left(\rho^{0}, \rho^{1}, \mu^{0}, \mu^{1}\right) \in \mathcal{X}^{\prime}$ obtained in Proposition 3 is such that

$\rho^{0}=\Psi(0), \rho^{1}=-\Psi_{t}(0)+V(0) \delta_{0}, \mu^{0}=-V(0), \mu^{1}=V_{t}(0)+\Psi(0,0)$.

By a density argument one can then deduce that the solution $(\Psi, V)$ obtained in Proposition 3 is such that the traces

$$
\left.\Psi\right|_{t=0},-\Psi_{t}+\left.V \delta_{0}\right|_{t=0},-\left.V\right|_{t=0}, V_{t}+\left.\Psi(0, t)\right|_{t=0}
$$

are well defined and coincide with $\left(\rho^{0}, \rho^{1}, \mu^{0}, \mu^{1}\right)$.

The same arguments allows us to show that the traces are also well defined at $t=T$. This suffices to assert that the weak solution of (28) we have constructed by transposition is at rest at $t=T$.

We can now complete the proof of Proposition 2.

End of the proof of Proposition 2. In view of Proposition 3 and Remark 4 we can define a linear and continuous map $\Lambda$ from $\mathcal{X}$ into $\mathcal{X}^{\prime}$ such that

$$
\Lambda\left(\eta^{0}, \eta^{1}, u^{0}, u^{1}\right)=\left(-\Psi_{t}+\left.V \delta_{0}\right|_{t=0}, \Psi(0), V_{t}+\left.\Psi(0, t)\right|_{t=0},-\left.V\right|_{t=0}\right) .
$$

Taking in (31), $\widetilde{f} \equiv 0, \widetilde{g} \equiv 0$ and $(\widetilde{\eta}, \widetilde{u})=(\eta, u)$, we deduce that

$$
\left\langle\Lambda\left(\eta^{0}, \eta^{1}, u^{0}, u^{1}\right),\left(\eta^{0}, \eta^{1}, u^{0}, u^{1}\right)\right\rangle=\int_{0}^{T} \rho(t)\left|u_{t t}(t)\right|^{2} d t
$$


and in view of (24) we deduce that there exists $C>0$ such that

$$
\left\langle\Lambda\left(\eta^{0}, \eta^{1}, u^{0}, u^{1}\right),\left(\eta^{0}, \eta^{1}, u^{0}, u^{1}\right)\right\rangle \geq C\left\|\left(\eta^{0}, \eta^{1}, u^{0} u^{1}\right)\right\|_{\mathcal{X}}^{2} .
$$

Actually, $C=(C(T, n))^{-1}$, where $C(T, n)$ is as in $(24)$.

This implies that $\Lambda$ is an isomorphism.

This shows that given any $\left(\rho^{1}, \rho^{0}, \mu^{1}, \mu^{0}\right) \in \mathcal{X}^{\prime}$ there exists

$$
\left(\eta^{0}, \eta^{1}, u^{0}, u^{1}\right)=\Lambda^{-1}\left(\rho^{1}, \rho^{0}, \mu^{1}, \mu^{0}\right)
$$

such that the corresponding solution of (28) in the sense of transposition satisfies

$$
\begin{aligned}
& \Psi(0)=\rho^{0},-\Psi_{t}+\left.V \delta_{0}\right|_{t=0}=\rho^{1} \\
& -\left.V\right|_{t=0}=\mu^{0}, V_{t}+\left.\Psi(0, t)\right|_{t=0}=\mu^{1}
\end{aligned}
$$

If we want this to be equivalent to the initial data of (19) we have to take

$$
\rho^{0}=\Psi^{0}, \rho^{1}=-\Psi^{1}+V^{0} \delta_{0}, \mu^{0}=-V^{0}, \mu^{1}=V^{1}+\Psi^{0}(0) .
$$

This makes sense when the data $\left(\Psi^{0}, \Psi^{1}, V^{0}, V^{1}\right)$ is in $\mathcal{X}$.

The control we have obtained is of the form

$$
\beta=-\frac{d^{2}}{d t^{2}}\left(\rho u_{t t}\right)
$$

where $u$ corresponds to the solution $(\eta, u)$ of $(27)$ with data

$$
\left(\eta^{0}, \eta^{1}, u^{0}, u^{1}\right)=\Lambda^{-1}\left(\rho^{1}, \rho^{0}, \mu^{1}, \mu^{0}\right),
$$

where $\left(\rho^{0}, \rho^{1}, \mu^{0}, \mu^{1}\right)$ is given by (34).

From the identities above we see that

$$
\begin{aligned}
\|\beta\|_{H^{-2}(0, T)}^{2} & \leq\left\|\rho u_{t t}\right\|_{L^{2}(0, T)}^{2} \\
& \leq C\left\|\left(\rho^{1}, \rho^{0}, \mu^{1}, \mu^{0}\right)\right\|_{\mathcal{X}^{\prime}}^{2} \\
& \leq C\left(\left\|\left(\Psi^{1}, \Psi^{0}, V^{1}, V^{0}\right)\right\|_{\mathcal{X}^{\prime}}^{2}+\left|\Psi^{0}(0)\right|^{2}\right),
\end{aligned}
$$

where $C=C(T, n)$ is the constant obtained in (24). 
REMARK 5. In fact, in some sense, we obtain a stronger result since we prove that we can control the problem (31) for any initial data $\left(\rho^{0}, \rho^{1}, \mu^{0}, \mu^{1}\right) \in \mathcal{X}^{\prime}$. In order to give an interpretation of the control problem in terms of the initial data $\left(\Psi^{1}, \Psi^{0}, V^{1}, V^{0}\right)$ we have to assure that $\Psi^{0}(0)$ makes sense. For this reason we consider that

$$
\left(\Psi^{1}, \Psi^{0}, V^{1}, V^{0}\right) \in \mathcal{X} .
$$

When $n=0$ one can not expect the same controllability result due to the conservation of the quantities (22) along the trajectories. In this case the controllability result reads as follows

Proposition 4. Assume that $T>2$ and $n=0$. Then, for any $\left(\Psi^{1}, \Psi^{0}, V^{1}, V^{0}\right) \in \mathcal{X}$ there exists a control $\beta \in H^{-2}(0, T)$ with compact support such that the solution $(\Psi, V)$ of (19) satisfies

$$
\begin{aligned}
& \Psi(T)=V^{1}+\Psi^{0}(0), \quad \Psi_{t}(T)=0, \\
& V(T)=V^{0}-\int_{0}^{1} \Psi^{1} d x, \quad V_{t}(T)=0 .
\end{aligned}
$$

REMARK 6. This result asserts that, when $n=0$, any solution of (19) can be driven to an equilibrium configuration which is a priori determined by the initial data.

Proof. First of all we observe that proving Proposition 4 is equivalent to showing that for any initial data as in the statement of Proposition 4 and satisfying the further assumptions

$$
V^{1}+\Psi^{0}(0)=0, \quad V^{0}-\int_{0}^{1} \Psi^{1}(x) d x=0,
$$

then, there exists a control $\beta$ such that

$$
\Psi(T)=\Psi_{t}(T) \equiv 0 \text { in }(0,1), \quad V(T)=V_{t}(T)=0 .
$$

Indeed, this is an immediate consequence of the remark made in the introduction that shows that when $\beta$ is of zero average the following identities hold

$$
\begin{aligned}
& V_{t}(T)+\Psi(0, T)=V^{1}+\Psi^{0}(0) \\
& V(T)-\int_{0}^{1} \Psi_{t}(x, T)=V^{0}-\int_{0}^{1} \Psi^{1}(x) .
\end{aligned}
$$


Thus, in the sequel we focus on initial data $\left(\Psi^{0}, \Psi^{1}, V^{0}, V^{1}\right)$ satisfying (39). For the adjoint system

$$
\begin{cases}\eta_{t t}-\eta_{x x}=0, & \text { in }(0,1) \times(0, T), \\ \eta_{x}(1)=0, & \text { for } t \in(0, T), \\ \eta_{x}(0)=u_{t}, & \text { for } t \in(0, T), \\ u_{t t}-\eta_{t}(0)=0, & \text { for } t \in(0, T) \\ \eta(0)=\eta^{0}, \eta_{t}(0)=\eta^{1}, & \text { in }(0,1) \\ u(0)=u^{0}, u_{t}(0)=u^{1}, & \end{cases}
$$

we consider initial data in the following subspace $\mathcal{X}_{0}$ of $\mathcal{X}$

(41) $\mathcal{X}_{0}=\left\{\left(\eta^{0}, \eta^{1}, u^{0}, u^{1}\right) \in \mathcal{X} u^{1}-\eta^{0}(0)=0, \int_{0}^{1} \eta^{1} d y+u^{0}=0\right\}$

It is easy to see that the subspace $\mathcal{X}_{0}$ is invariant under the flow generated by (40).

Given $\left(\eta^{0}, \eta^{1}, u^{0}, u^{1}\right) \in \mathcal{X}_{0}$ we solve first (40) and then the backward system

$$
\begin{cases}\Psi_{t t}-\Psi_{x x}=0, & \text { in }(0,1) \times(0, T), \\ \Psi_{x}(1, t)=0, & \text { for } t \in(0, T), \\ \Psi_{x}(0, t)=-V_{t}(t), & \text { for } t \in(0, T), \\ V_{t t}(t)+\Psi_{t}(0, t)=-\frac{d^{2}}{d t^{2}}\left(\rho(t) u_{t t}(t)\right), & \text { for } t \in(0, T), \\ \Psi(T)=\Psi_{t}(T)=0, & \text { in }(0,1), \\ V(T)=V_{t}(T)=0, & \end{cases}
$$

where $\rho$ is as in the proof of Proposition 2 .

Proceeding as in the proof of Proposition 3 one can show that (42) has a unique solution defined by transposition such that the traces (38) are well defined. On the other hand, integrating the equations in (42) we deduce that

$$
\int_{0}^{1} \rho^{1}(x) d x=0, \quad \mu^{1}=0 .
$$


Let us denote by $Z$ the subspace of $\mathcal{X}^{\prime}$ satisfying (43). More precisely,

$$
Z=\left\{\left(\rho^{1}, \rho^{0}, \mu^{1}, \mu^{0}\right) \in \mathcal{X}^{\prime} \text { such that (43) holds }\right\} \text {. }
$$

It is easy to check that $Z$ is actually the dual of $\mathcal{X}_{0}$. Indeed, the dual of $\mathcal{X}_{0}$ is a quotient space of $\mathcal{X}^{\prime}$ and there is a one-to-one correspondence between $Z$ and this quotient space in the sense that, in $Z$, we have chosen the unique element of each class of the quotient space satisfying (43).

As in the proof of Proposition 2 we can define a linear and continuous operator $\Lambda: \mathcal{X}_{0} \longrightarrow Z$ that associates the trace $\left(\rho^{1}, \rho^{0}, \mu^{1}, \mu^{0}\right) \in Z$ in $(31)$ to each $\left(\eta^{0}, \eta^{1}, u^{0}, u^{1}\right) \in \mathcal{X}_{0}$.

We also have

$$
\left\langle\Lambda\left(\eta^{0}, \eta^{1}, u^{0}, u^{1}\right),\left(\eta^{0}, \eta^{1}, u^{0}, u^{1}\right)\right\rangle=\int_{0}^{T} \rho(t)\left|u_{t t}(t)\right|^{2} d t .
$$

Remark that inequality (24) also holds for the case $n=0$ if we consider initial data in $\mathcal{X}_{0}$. Hence there exists a constant $C>0$ such that

$$
\left\langle\Lambda\left(\eta^{0}, \eta^{1}, u^{0}, u^{1}\right),\left(\eta^{0}, \eta^{1}, u^{0}, u^{1}\right)\right\rangle \geq C\left\|\left(\eta^{0}, \eta^{1}, u^{0}, u^{1}\right)\right\|_{\mathcal{X}^{\prime}}^{2},
$$

for all $\left(\eta^{0}, \eta^{1}, u^{0}, u^{1}\right) \in \mathcal{X}_{0}$, since the quantity $\left(\left\|\eta_{x}^{0}\right\|_{L^{2}(0,1)}^{2}+\left\|\eta^{1}\right\|_{L^{2}(0,1)}^{2}+\right.$ $\left.\left|u^{1}\right|^{2}\right)^{1 / 2}$ defines a norm in $\mathcal{X}_{0}$ which is equivalent to the norm induced by $\mathcal{X}$.

We deduce that $\Lambda: \mathcal{X}_{0} \longrightarrow Z$ is an isomorphism.

Then, given initial data as in the statement of Proposition 4 and such that (37) holds we define $\left(\rho^{1}, \rho^{0}, \mu^{1}, \mu^{0}\right) \in Z$ by (35). The control we are looking for is

$$
\beta=-\frac{d^{2}}{d t^{2}}\left(\rho(t) u_{t t}(t)\right)
$$

where $u$ is the second component of the solution $(\eta, u)$ of (40) with initial data $\left(\eta^{0}, \eta^{1}, u^{0}, u^{1}\right)=\Lambda^{-1}\left(\rho^{1}, \rho^{0}, \mu^{1}, \mu^{0}\right)$.

This concludes the proof of the Proposition.

Let us now state the controllability results for the two-dimensional system (4).

We use the Fourier decomposition method described at the beginning of this section. Thus we develop the initial data $\left(\Phi^{0}, \Phi^{1}, W^{0}, W^{1}\right)$ 
to be controlled in Fourier series

$$
\begin{cases}\Phi^{0}=\sum_{n=0}^{\infty} \Psi_{n}^{0}(x) \cos (n \pi y), & \Phi^{1}=\sum_{n=0}^{\infty} \Psi_{n}^{1}(x) \cos (n \pi y), \\ W^{0}=\sum_{n=0}^{\infty} V_{n}^{0} \cos (n \pi y), & W^{1}=\sum_{n=0}^{\infty} V_{n}^{1} \cos (n \pi y) .\end{cases}
$$

We assume that for every $n=0,1, \ldots$ the initial data satisfy the assumptions of Proposition 2 and Proposition 4. We set

$$
\rho_{n}^{0}=\Psi_{n}^{0}, \rho_{n}^{1}=-\Psi_{n}^{1}+V_{n}^{0} \delta_{0}, \mu^{0}=-V_{n}^{0}, \mu_{n}^{1}=V_{n}^{1}+\Psi_{n}^{0}(0) .
$$

We introduce the following space of initial data

$$
\begin{array}{r}
H=\left\{\left(\Phi^{0}, \Phi^{1}, W^{0}, W^{1}\right) \in \mathcal{Y}: \sum_{n=0}^{\infty} C(n, T)\left\|\left(\rho_{n}^{1}, \rho_{n}^{0}, \mu_{n}^{1}, \mu_{n}^{0}\right)\right\|_{\mathcal{X}^{\prime}}^{2}\right. \\
\left.=\left\|\left(\Phi^{0}, \Phi^{1}, W^{0}, W^{1}\right)\right\|_{H}^{2}<\infty\right\} .
\end{array}
$$

where the constants $C(n, T)$ are those appearing in (24).

Proposition 5. Assume that $T>2$. Then, for every initial data $\left(\Phi^{0}, \Phi^{1}, W^{0}, W^{1}\right)$ in $H$ there exists a control $\beta \in H^{-2}\left(0, T ; L^{2}(0,1)\right)$ such that the solution $(\Phi, W)$ of (4) satisfies

$$
\left\{\begin{aligned}
\Phi(T) & \equiv \mu^{1}=\int_{0}^{1} W^{1}(y) d y+\int_{0}^{1} \Psi^{0}(0, y) d y, \Phi_{t}(T) \equiv 0 \\
W(T) & \equiv\left\langle\rho^{1}, 1\right\rangle \\
& =\int_{0}^{1} W^{0}(y) d y-\int_{0}^{1} \int_{0}^{1} \Psi^{1}(x, y) d x d y, W_{t}(T) \equiv 0 .
\end{aligned}\right.
$$

Moreover there exists a constant $C>0$ such that

$$
\|\beta\|_{H^{-2}\left(0, T ; L^{2}(0,1)\right)} \leq C\left\|\left(\Phi^{0}, \Phi^{1}, W^{0}, W^{1}\right)\right\|_{H} .
$$

REMARK 7 . The control time $T>2$ is optimal. Indeed, when $T<2$ it is easy to see that the set of controllable data is not dense in the space of finite energy data. Actually, when $T<2$ none of the onedimensional problems (19) is approximately controllable, i.e. the space of controllable data is no even dense in $\mathcal{Y}^{\prime}$. 
REMARK 8 . The constants $C(n, T)$ play an important role in the controllability problem since the space $H$ of controllable functions depends on them. The next two sections are devoted to the evaluation of these constants.

Proof. In view of propositions 2 and 4 for any $n=0,1, \ldots$ there exists a control $\beta_{n} \in H^{-2}(0, T)$ such that the solution $\left(\Psi_{n}, V_{n}\right)$ of (19) satisfies

$$
\Psi_{n}(T) \equiv \Psi_{n, t}(T)=0 \text { in }(0,1), \quad V_{n}(T)=V_{n, t}(T)=0,
$$

for $n \geq 1$ and

$$
\begin{aligned}
& \Psi_{0}(T)=\mu^{1}, \Psi_{0, t}(T)=0 \text { in }(0,1), \\
& V_{0}(T)=\left\langle\rho^{1}, 1\right\rangle, V_{0, t}(T)=0
\end{aligned}
$$

when $n=0$.

On the other hand

$$
\left\|\beta_{n}\right\|_{H^{-2}(0, T)}^{2} \leq C(n, T)\left\|\left(\rho_{n}^{1}, \rho_{n}^{0}, \mu_{n}^{1}, \mu_{n}^{0}\right)\right\|_{\mathcal{X}^{\prime}}^{2}
$$

We construct the following control for the two-dimensional system

$$
\beta(y, t)=\sum_{n=0}^{\infty} \beta_{n} \cos (n \pi y) .
$$

We have, in view of (52),

$$
\begin{aligned}
\|\beta\|_{H^{-2}\left(0, T ; L^{2}(0,1)\right)}^{2} & =\sum_{n=0}^{\infty}\left\|\beta_{n}(t)\right\|_{H^{-2}(0, T)}^{2} \\
& \leq \sum_{n=0}^{\infty} C(n, T)\left\|\left(\rho_{n}^{1}, \rho_{n}^{0}, \mu_{n}^{1}, \mu_{n}^{0}\right)\right\|_{\mathcal{X}^{\prime}}^{2} \\
& =\left\|\left(\Psi^{0}, \Psi^{1}, W^{0}, W^{1}\right)\right\|_{H}^{2} \\
& <\infty
\end{aligned}
$$

Therefore $\beta \in H^{-2}\left(0, T ; L^{2}(0,1)\right)$. On the other hand,

$$
\Psi(x, y, t)=\sum_{n=0}^{\infty} \Psi_{n}(y, t) \cos (n \pi y), \quad W(y, t)=\sum_{n=0}^{\infty} V_{n}(t) \cos (n \pi y)
$$


solves (4) with the control $\beta$ given in (53) and satisfies (48) at time $t=T$.

This concludes the proof of this Proposition.

\section{Spectral analysis.}

In this section we give some estimates on the spectrum of the differential operator corresponding to (23) that will be used in the next section to prove (24). In order to analyze the spectrum of (23) let $(\Psi(x, t), V(t))$ be solution of

$$
\begin{cases}\Psi_{t t}-\Psi_{x x}+n^{2} \pi^{2} \psi=0, & \text { in }(0,1) \times(0, \infty) \\ \Psi_{x}(1)=0, & \text { for } t \in(0, \infty), \\ \Psi_{x}(0)=V_{t}, & \text { for } t \in(0, \infty) \\ V_{t t}+n^{4} \pi^{4} V-\psi_{t}(0)=0, & \text { for } t \in(0, \infty)\end{cases}
$$

Now if we look for solutions of (54) of the form $(\Psi(x, t), V(t))=$ $e^{\lambda t}(\Psi(x), V)$, with $V \in \mathbb{R}$, it follows that the eigenvalues $\lambda$ of system (1) are the roots of the equation

$$
e^{2 \sqrt{\lambda^{2}+n^{2} \pi^{2}}}=-\frac{\lambda^{2}-\sqrt{\lambda^{2}+n^{2} \pi^{2}}\left(\lambda^{2}+n^{4} \pi^{4}\right)}{\lambda^{2}+\sqrt{\lambda^{2}+n^{2} \pi^{2}}\left(\lambda^{2}+n^{4} \pi^{4}\right)} .
$$

We have the following first result

Lemma 1. System (1) has a two-parameter sequence of purely imaginary eigenvalues $\left\{\lambda_{n, k}\right\}_{n \in \mathbb{N}, k \in \mathbb{Z}^{*}}$ given by

$$
\lambda_{n, k}=\sqrt{z_{n, k}^{2}+n^{2} \pi^{2}} i
$$

if $k>0$ and $\lambda_{n, k}=-\lambda_{n,-k}$ if $k<0$, where $\left\{z_{n, k}\right\}_{k \in \mathbb{N}^{*}}$ are the roots of the equation

$$
\tan z=\frac{z^{2}+n^{2} \pi^{2}}{z^{3}+z\left(n^{2} \pi^{2}-n^{4} \pi^{4}\right)} .
$$

Moreover, there are another two eigenvalues of (1), $\lambda_{n}^{*}$ and $\lambda_{n}^{* *}$, with the modulus less than $n \pi$, given by

$$
\lambda_{n}^{*}=\sqrt{n^{2} \pi^{2}-\left(z_{n}^{*}\right)^{2}} i, \quad \lambda_{n}^{* *}=\bar{\lambda}_{n}^{*},
$$


where $z_{n}^{*}$ is the unique positive root of the equation

$$
e^{2 z}=\frac{z^{3}-z^{2}+n^{2} \pi^{2}+z\left(n^{4} \pi^{4}-n^{2} \pi^{2}\right)}{z^{3}+z^{2}-n^{2} \pi^{2}+z\left(n^{4} \pi^{4}-n^{2} \pi^{2}\right)}
$$

In the last case, $\lambda_{n}^{*}=\lambda_{n}^{* *}=0$ when $n=0$.

Proof. We know that the eigenvalues $\lambda$ are roots of (55). Considering the change of variable $\lambda=\sqrt{\zeta^{2}-n^{2} \pi^{2}}$ equation (55) becomes

$$
e^{2 \zeta}=\frac{\zeta^{3}-\zeta^{2}+n^{2} \pi^{2}+\zeta\left(n^{4} \pi^{4}-n^{2} \pi^{2}\right)}{\zeta^{3}+\zeta^{2}-n^{2} \pi^{2}+\zeta\left(n^{4} \pi^{4}-n^{2} \pi^{2}\right)}
$$

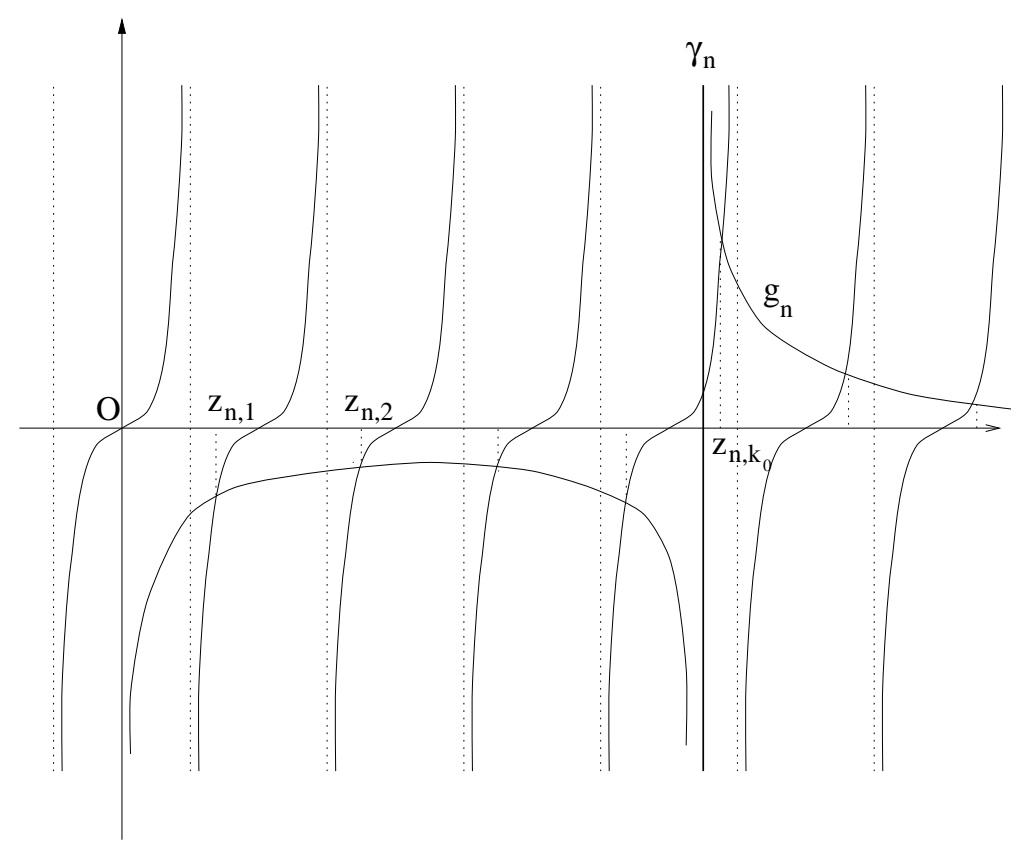

Figure 1.

Since the differential operator corresponding to (1) is conservative its eigenvalues will be all purely imaginary. Hence, we have to look only for those roots of (60) which are purely imaginary or real. It follows that the imaginary roots of (60) are the roots of the equation (57) and the real ones are roots of (59). 


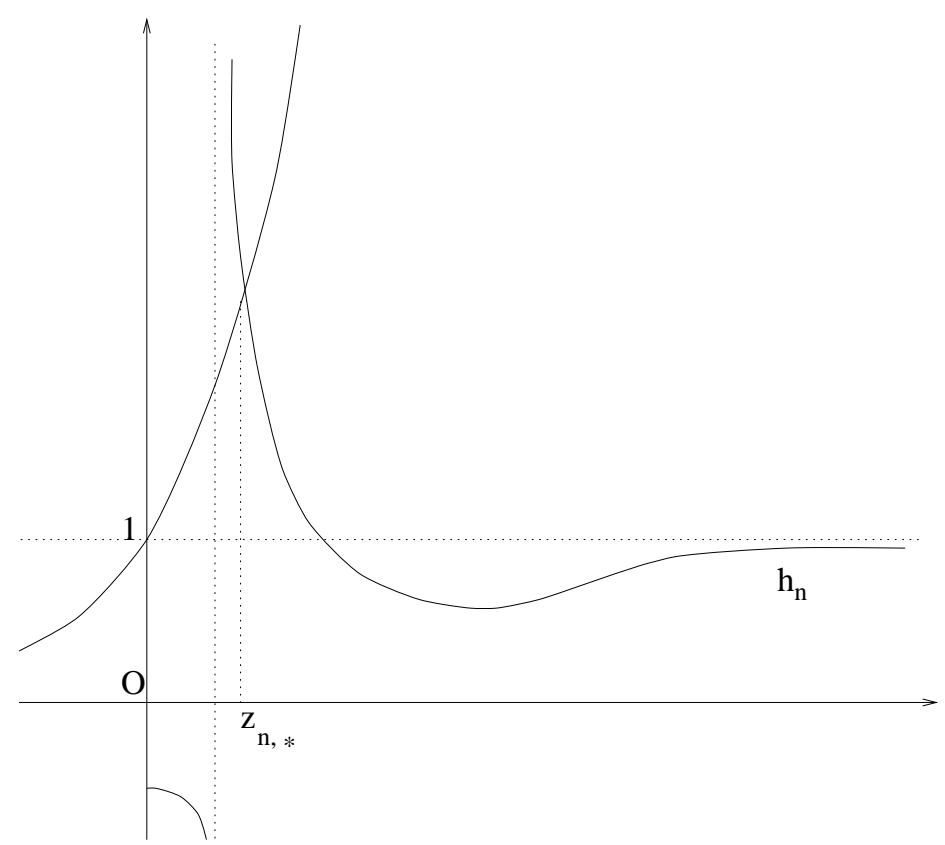

Figure 2.

Observe that the right hand side of (57) has a pole at

$$
z=\sqrt{n^{4} \pi^{4}-n^{2} \pi^{2}} .
$$

Let us denote by $\alpha_{n}=n^{4} \pi^{4}-n^{2} \pi^{2}, \gamma_{n}=\sqrt{\alpha_{n}}$ and let $k_{0} \in \mathbb{N}$ be such that $k_{0} \pi-\pi / 2 \leq \sqrt{\alpha_{n}}<k_{0} \pi+\pi / 2$.

Equation (57) has an unique root in each interval $(k \pi-\pi / 2, k \pi+$ $\pi / 2)$ for $k \in \mathbb{N} \backslash\left\{k_{0}\right\}$. (57).

In $\left(k_{0} \pi-\pi / 2, k_{0} \pi+\pi / 2\right)$ there are two roots $z_{n, k_{0}-1}$ and $z_{n, k_{0}}$ of

The localization of the roots $\left\{z_{k, m}\right\}_{k \in \mathbb{N}^{*}}$ and $z_{n, *}$ is illustrated in figures 1 and 2, where

$$
g_{n}(z)=\frac{z^{2}+n^{2} \pi^{2}}{z^{3}+z\left(n^{2} \pi^{2}-n^{4} \pi^{4}\right)}
$$

and

$$
h_{n}(z)=\frac{z^{3}-z^{2}+n^{2} \pi^{2}+z\left(n^{4} \pi^{4}-n^{2} \pi^{2}\right)}{z^{3}+z^{2}-n^{2} \pi^{2}+z\left(n^{4} \pi^{4}-n^{2} \pi^{2}\right)} .
$$

The roots correspond to the points of intersection of the curves in the figures. 
The skew adjoint operator corresponding to (1) can be diagonalised over the orthogonal basis of eigenvectors

$$
\begin{aligned}
\xi_{n, k} & =\left(\begin{array}{l}
\xi_{n, k}^{1} \\
\xi_{n, k}^{2} \\
\xi_{n, k}^{3} \\
\xi_{n, k}^{4}
\end{array}\right) \\
= & \left(\begin{array}{l}
\frac{1}{\lambda_{n, k}} \cosh \left(\sqrt{n^{2} \pi^{2}+\lambda_{n, k}^{2}}(x-1)\right) \cos (n \pi y) \\
\frac{-\cosh \left(\sqrt{n^{2}} \pi^{2}+\lambda_{n, k}^{2}\right.}{n^{2} \pi^{2}+\lambda_{n, k}^{2}} \sinh \left(\sqrt{n^{2} \pi^{2}+\lambda_{n, k}^{2}}\right) \cos (n \pi y) \\
\lambda_{n, k}^{2} \\
\frac{\sqrt{n^{2} \pi^{2}+\lambda_{n, k}^{2}}}{\lambda_{n, k}} \sinh \left(\sqrt{n^{2} \pi^{2}+\lambda_{n, k}^{2}}\right) \cos (n \pi y)
\end{array}\right)
\end{aligned}
$$

and the solution of (5) with initial condition $\xi_{n, k}$ is such that

$$
\left(\begin{array}{c}
\Psi(x, y, t) \\
\Psi_{t}(x, y, t) \\
W(y, t) \\
W_{t}(y, t)
\end{array}\right)=\xi_{n, k}(x, y) e^{\lambda_{n, k} t}
$$

As this basis is not normalized, we will denote $\Xi_{n, k}=\left\|\xi_{n, k}\right\|_{\mathcal{Y}}$. Notice that if $n$ and $k$ are integers,

$$
c \leq \Xi_{n, k} \leq C
$$

On the other hand $z_{n, *}$ is the only positive real solution to

$$
e^{2 t}=h_{n}(t)=\frac{N(t)}{D(t)}=\frac{-t^{2}+n^{2} \pi^{2}+t\left(t^{2}+n^{4} \pi^{4}-n^{2} \pi^{2}\right)}{t^{2}-n^{2} \pi^{2}+t\left(t^{2}+n^{4} \pi^{4}-n^{2} \pi^{2}\right)} .
$$

Let $t_{0}(n)$ be the real root of $D$. It follows that $z_{n, *}>t_{0}(n)>n^{-3}$. Furthermore, as $D\left(n^{1 / 2}\right)>0$ and $R\left(n^{1 / 2}\right) \sim 1<e^{2 n^{1 / 2}}, z_{n, *}<n^{1 / 2}$ for large $n$. 
Therefore, as $\lambda_{n, *}=i \sqrt{n^{2} \pi^{2}-z_{n, *}^{2}}$,

$$
c n^{-8} \leq\left|\frac{\lambda_{n, *}}{i n}-\pi\right| \leq C n^{-1}
$$

and

$$
c n^{-3} \leq \Xi_{n, *} \leq C e^{n^{2 / 3}} .
$$

For any $\left(\Phi^{0}, \Phi^{1}, W^{0}, W^{1}\right)$ in $\mathcal{Y}$,

$$
\left(\Phi^{0}, \Phi^{1}, W^{0}, W^{1}\right)=\sum_{\substack{n \in \mathbb{N} \\ k \in \mathbb{Z}^{*} \cup\{*, * *\}}} \frac{a_{n, k}}{\Xi_{n, k}} \xi_{n, k}(x, y),
$$

with $\left\{a_{n, k}\right\}_{n, k} \in l^{2}$.

Let us now make some notations. We will write for any $\left(\Phi^{0}, \Phi^{1}, W^{0}, W^{1}\right)$ in $\mathcal{Y}$ that

- $\left(\Phi^{0}, \Phi^{1}, W^{0}, W^{1}\right) \in \mathcal{Y}^{n_{0}}$ if $n \neq n_{0}$ implies $a_{n, k}=0$,

- $\left(\Phi^{0}, \Phi^{1}, W^{0}, W^{1}\right) \in \mathcal{Y}^{(1)}$ if $|k|>|n|$ implies $a_{n, k}=0$,

0 ,

- $\left(\Phi^{0}, \Phi^{1}, W^{0}, W^{1}\right) \in \mathcal{Y}^{(2)}$ if $|k| \leq|n|$ or $k \in\{*, * *\}$ implies $a_{n, k}=$

- $\left(\Phi^{0}, \Phi^{1}, W^{0}, W^{1}\right) \in \mathcal{Y}^{i, n}$ implies $\left(\Phi^{0}, \Phi^{1}, W^{0}, W^{1}\right) \in \mathcal{Y}^{(i)} \cap \mathcal{Y}^{n}$.

We can denote

$$
\left(\Phi^{0}, \Phi^{1}, W^{0}, W^{1}\right)=\left(\Phi^{0}, \Phi^{1}, W^{0}, W^{1}\right)^{(1)}+\left(\Phi^{0}, \Phi^{1}, W^{0}, W^{1}\right)^{(2)},
$$

with $\left(\Phi^{0}, \Phi^{1}, W^{0}, W^{1}\right)^{(i)} \in \mathcal{Y}^{(i)}$.

Moreover $I$ will be the set of $(k, n)$ such that $k \in\{*, * *\}$ or $|k| \leq$ $|n|$, and we will agree that $* *=-*$. To end with, we shall also denote $\nu_{n, k}=\operatorname{Im} \lambda_{n, k}$ to deal with real numbers.

\section{Proof of Theorem 1.}

In order to prove the theorem, we will use a proposition for low frequencies and a lemma for high ones. 
Proposition 6 (Low frequencies). For any positive $\varepsilon$ and $\delta$, there exists a constant $C_{\varepsilon, \delta}$, an integer $n_{1}(\varepsilon)$ and a positive time $T_{2}(\varepsilon, \delta) \leq C_{\delta} / \varepsilon^{1+\delta}$ such that for any integer $n$ greater than $n_{1}(\varepsilon)$ and any $\left(\Phi^{0}, \Phi^{1}, W^{0}, W^{1}\right)$ in $\mathcal{Y}^{n}$, the solution of (5) with initial condition $\left(\Phi^{0}, \Phi^{1}, W^{0}, W^{1}\right)$ satisfies

$$
\left\|\left(\Phi^{0}, \Phi^{1}, W^{0}, W^{1}\right)^{(1)}\right\|_{\mathcal{Y}}^{2} \leq C_{\varepsilon, \delta} e^{2 \varepsilon|n|} \int_{-T_{2}(\varepsilon, \delta)}^{T_{2}(\varepsilon, \delta)}\left|W_{t t}(0, t)\right|^{2} d t
$$

This proposition will be proved in Section 4.2.

Lemma 2 (High frequencies). There exists a constant $C$ and a positive time $T_{1}$ such that for integer $n$ and any $\left(\Phi^{0}, \Phi^{1}, W^{0}, W^{1}\right)$ in $\mathcal{Y}^{n, 2}$, the solution of (5) with initial condition $\left(\Phi^{0}, \Phi^{1}, W^{0}, W^{1}\right)$ satisfies

$$
\left\|\left(\Phi^{0}, \Phi^{1}, W^{0}, W^{1}\right)\right\|_{\mathcal{Y}} \leq \frac{C}{n^{4}}\left\|W_{t t}\right\|_{L^{2}\left(\left(0, T_{1}\right) \times \Gamma_{0}\right)} .
$$

The proof of Lemma 2 will be given in subsection 4.1.

Let us now prove how do Proposition 6 and Lemma 2 imply that Theorem 1 is true.

Proof of Theorem 1 . Let $\varepsilon$ and $\delta$ be two positive real numbers. Out of Propositions 6 and Lemma 6 , we get two positive times, denoted $T_{1}$ and $T_{2}(\varepsilon, \delta)$. Let us define $T(\varepsilon, \delta)=\sup \left\{T_{1}, T_{2}(\varepsilon, \delta)\right\}$.

Let $n$ be a positive integer and $\left(\Phi^{0}, \Phi^{1}, W^{0}, W^{1}\right)$ any initial condition in $\mathcal{Y}^{n}$. Then we have

$$
\begin{aligned}
\left\|\left(\Phi^{0}, \Phi^{1}, W^{0}, W^{1}\right)\right\|_{\mathcal{Y}}^{2} & \\
& =\left\|\left(\Phi^{0}, \Phi^{1}, W^{0}, W^{1}\right)^{(1)}\right\|_{\mathcal{Y}}^{2}+\left\|\left(\Phi^{0}, \Phi^{1}, W^{0}, W^{1}\right)^{(2)}\right\|_{\mathcal{Y}}^{2} .
\end{aligned}
$$

So by Proposition 6 and Lemma 2, for $n \geq n_{1}(\varepsilon)$,

$$
\begin{aligned}
\left\|\left(\Phi^{0}, \Phi^{1}, W^{0}, W^{1}\right)\right\|_{\mathcal{Y}}^{2} \leq & C_{\varepsilon, \delta} e^{2 \varepsilon|n|} \int_{-T_{2}(\varepsilon, \delta)}^{T_{2}(\varepsilon, \delta)}\left|W_{t t}(0, t)\right|^{2} d t \\
& +\frac{C}{n^{4}} \int_{0}^{T_{1}}\left|W^{(2)} t t(0, t)\right|^{2} d t \\
\leq & C_{\varepsilon, \delta} e^{2 \varepsilon|n|} \int_{-T(\varepsilon, \delta)}^{T(\varepsilon, \delta)}\left|W_{t t}(0, t)\right|^{2} d t
\end{aligned}
$$




$$
\begin{aligned}
& +\frac{C}{n^{4}} \int_{0}^{T_{1}}\left|W_{t t}(0, t)\right|^{2} d t \\
& +\frac{C}{n^{4}} \int_{0}^{T_{1}}\left|W^{(1)}{ }_{t t}(0, t)\right|^{2} d t .
\end{aligned}
$$

Therefore, by the direct estimate (14),

$$
\begin{aligned}
& \left\|\left(\Phi^{0}, \Phi^{1}, W^{0}, W^{1}\right)\right\|_{\mathcal{Y}}^{2} \\
& \quad \leq C_{\varepsilon, \delta}^{\prime} e^{2 \varepsilon|n|} \int_{-T(\varepsilon, \delta)}^{T(\varepsilon, \delta)}\left|W_{t t}(0, t)\right|^{2} d t+C^{\prime}\left\|\left(\Phi^{0}, \Phi^{1}, W^{0}, W^{1}\right)^{(1)}\right\|_{\mathcal{Y}}^{2} .
\end{aligned}
$$

So by Proposition 6,

$$
\left\|\left(\Phi^{0}, \Phi^{1}, W^{0}, W^{1}\right)\right\|_{\mathcal{Y}}^{2} \leq C_{\varepsilon, \delta}^{\prime} e^{2 \varepsilon|n|} \int_{-T(\varepsilon, \delta)}^{T(\varepsilon, \delta)}\left|W_{t t}(0, t)\right|^{2} d t .
$$

We can increase the constant to take care of the first $n_{1}(\varepsilon)$ values of $n$. As $T(\varepsilon, \delta) \leq T_{2}(\varepsilon, \delta) \leq C / \varepsilon^{1+\delta}$, if we put

$$
T(\alpha(T), \delta)=T
$$

we get

$$
\alpha(T) \leq \frac{C_{q}}{T^{1-q}},
$$

for any positive real number $q$ and (7) is proved.

We pass now to prove Lemma 2 .

\subsection{Proof of Lemma 2 .}

Since $\left(\Phi^{0}, \Phi^{1}, W^{0}, W^{1}\right) \in \mathcal{Y}^{2, n}$,

$$
\begin{aligned}
& \left(\Phi^{0}, \Phi^{1}, W^{0}, W^{1}\right)=\sum_{|k|>n} a_{n, k} \frac{\xi_{n, k}}{\Xi_{n, k}}, \\
& \left\|\left(\Phi^{0}, \Phi^{1}, W^{0}, W^{1}\right)^{(2)}\right\|_{\mathcal{Y}}^{2}=\sum_{|k|>n}\left|a_{n, k}\right|^{2} .
\end{aligned}
$$


On the other hand, for $T_{1}>0$,

$$
\begin{aligned}
& \int_{0}^{T_{1}}\left|W_{t t}(t, 0)\right|^{2} \\
& =\int_{0}^{T_{1}}\left|\nu_{n, k}^{2} \sum_{|k|>n} a_{n, k} \frac{\xi_{n, k}^{3}}{\Xi_{n, k}} e^{\nu_{n, k} t i}\right|^{2} \\
& =\int_{0}^{T_{1}}\left|\sum_{|k|>n} \frac{a_{n, k}}{\Xi_{n, k}} \sqrt{n^{2} \pi^{2}-\nu_{n, k}^{2}} \sin \left(\sqrt{n^{2} \pi^{2}-\nu_{n, k}^{2}}\right) e^{\nu_{n, k} t i}\right|^{2} .
\end{aligned}
$$

Let us prove that there exists $c>0$ such that, for $k>n$,

$$
\nu_{n, k+1}-\nu_{n, k} \geq c .
$$

Firstly, remark that $z_{n, k+1}-z_{n, k}>\pi / 2$, for all $k \neq k_{0}-1, k_{0}$ where $k_{0} \in \mathbb{N}$ is such that $\left(k_{0}-1\right) \pi+\pi / 2 \leq \sqrt{\alpha_{n}}<k_{0} \pi+\pi / 2$. We recall that $\alpha_{n}=n^{4} \pi^{4}-n^{2} \pi^{2}$. In order to prove that there is a gap between $z_{n, k_{0}-1}$ and $z_{n, k_{0}}$ let us show that, if $z \in\left(\left(k_{0}-1\right) \pi+\pi / 2, k_{0} \pi+\pi / 2\right)$ then

$$
\left|\frac{z^{2}+n^{2} \pi^{2}}{z^{3}-z \alpha_{n}}\right| \geq \frac{1}{\pi}
$$

Indeed we have

$$
\begin{aligned}
& \left|\frac{z^{2}+n^{2} \pi^{2}}{z^{3}-z \alpha_{n}}\right| \\
& \geq \min \left\{\left|\frac{\left(\left(k_{0}-1\right) \pi+\frac{\pi}{2}\right)^{2}+n^{2} \pi^{2}}{\left(\left(k_{0}-1\right) \pi+\frac{\pi}{2}\right)^{3}-\left(\left(k_{0}-1\right) \pi+\frac{\pi}{2}\right) \alpha_{n}}\right|,\right. \\
& \left.\geq \frac{\left(\left(k_{0}+1\right) \pi+\frac{\pi}{2}\right)^{2}+n^{2} \pi^{2}}{\alpha_{n}+\sqrt{\alpha_{n} \sqrt{\alpha_{n}}}} \mid\right\} \\
& \cdot \min \left\{\frac{1}{\left(\left(k_{0}+1\right) \pi+\frac{\pi}{2}\right)^{3}-\left(\left(k_{0}+1\right) \pi+\frac{\pi}{2}\right) \alpha_{n}} \mid\right\} \\
& \geq \frac{1}{\pi} \cdot \quad \frac{1}{\left|\left(k_{0}+1\right) \pi+\frac{\pi}{2}-\sqrt{\alpha_{n}}\right|}\left|\frac{\left(k_{0}-1\right) \pi+\frac{\pi}{2}-\sqrt{\alpha_{n}} \mid}{\alpha^{2}}\right|
\end{aligned}
$$


From (67) it follows that $\max \left\{\left|\tan z_{n, k_{0}-1}\right|,\left|\tan z_{n, k_{0}}\right|\right\}>1 / \pi$.

Hence $\left|z_{n, k_{0}}-z_{n, k_{0}-1}\right|>\arctan (1 / \pi)$.

We can evaluate now

$$
\begin{aligned}
\nu_{n, k+1}-\nu_{n, k} & =\sqrt{n^{2} \pi^{2}+z_{n, k+1}^{2}}-\sqrt{n^{2} \pi^{2}+z_{n, k}^{2}} \\
& >\frac{\left(z_{n, k+1}-z_{n, k}\right)\left(z_{n, k+1}+z_{n, k}\right)}{2 \sqrt{n^{2} \pi^{2}+z_{n, k}^{2}}} \\
& >\arctan \frac{1}{\pi} \frac{n \pi}{4 n \pi} \\
& =\frac{1}{4} \arctan \frac{1}{\pi}
\end{aligned}
$$

and (66) holds with $c=(1 / 4) \arctan (1 / \pi)$.

By using Ingham's inequality (see Ingham [5]) we obtain that, for $T_{1}>2 \pi / c$,

$$
\begin{aligned}
& \int_{0}^{T_{1}}\left|W_{t t}(t, 0)\right|^{2} \\
& \quad \geq C \sum_{|k|>n}\left|\frac{a_{n, k}}{\Xi_{n, k}}\right|^{2}\left|\sqrt{n^{2} \pi^{2}-\nu_{n, k}^{2}} \sin \left(\sqrt{n^{2} \pi^{2}-\nu_{n, k}^{2}}\right)\right|^{2} .
\end{aligned}
$$

Let us prove that

$$
\left|\sqrt{n^{2} \pi^{2}-\nu_{n, k}^{2}} \sin \left(\sqrt{n^{2} \pi^{2}-\nu_{n, k}^{2}}\right)\right|=\left|z_{n, k} \sin z_{n, k}\right| \geq \frac{C}{n^{4}},
$$

where $C$ is a positive constant not depending on $n$ and $k$.

Firstly, from (57), we have

$$
z_{n, k} \sin z_{n, k}=\frac{z_{n, k}^{2}+n^{2} \pi^{2}}{z_{n, k}^{2}+\alpha_{n}} \cos z_{n, k} .
$$

Consider the following cases

i) $z_{n, k}>\sqrt{\alpha_{n}}$. In this case

$$
\frac{z_{n, k}^{2}+n^{2} \pi^{2}}{z_{n, k}^{2}-\alpha_{n}}>\frac{z_{n, k}\left(z_{n, k}-\sqrt{\alpha_{n}}\right)}{z_{n, k}^{2}-\alpha_{n}}=\frac{1}{1+\frac{\sqrt{\alpha_{n}}}{z_{n, k}}}>\frac{C}{n^{2}} .
$$


If $\left|\cos z_{n, k}\right| \geq 1 / \sqrt{2}$ then

$$
z_{n, k} \sin z_{n, k}=\frac{z_{n, k}^{2}+n^{2} \pi^{2}}{z_{n, k}^{2}+\alpha_{n}} \cos z_{n, k}>\frac{C}{n^{2}} .
$$

If $\left|\cos z_{n, k}\right|<1 / \sqrt{2}$ then $\left|\sin z_{n, k}\right| \geq 1 / \sqrt{2}$ and

$$
\left|z_{n, k} \sin z_{n, k}\right| \geq \frac{z_{n, k}}{\sqrt{2}}>\frac{\sqrt{\alpha_{n}}}{\sqrt{2}}
$$

ii) $z_{n, k}<\sqrt{\alpha_{n}}$. Now we have

$$
\left|\tan z_{n, k}\right|>\inf _{z<\sqrt{\alpha_{n}}} \frac{z_{n, k}^{2}+n^{2} \pi^{2}}{z_{n, k}^{3}+z_{n, k} \alpha_{n}}>\frac{1}{n^{6}} .
$$

It follows that

$$
\left|z_{n, k} \sin z_{n, k}\right| \geq \frac{C}{n^{4}}
$$

Finally, we obtain that (69) holds.

From (65), (68) and (61) it follows that

$$
\left\|\left(\Phi^{0}, \Phi^{1}, W^{0}, W^{1}\right)\right\|_{\mathcal{Y}}^{2} \leq \frac{C}{n^{4}} \int_{0}^{T_{1}}\left|W_{t t}(t, 0)\right|^{2} .
$$

We still have to prove Proposition 6. This will be dealt with in the following subsection.

\subsection{Proof of Proposition 6.}

This proposition deals with the lowest eigenmodes of the problem. In this part of the spectrum, the Ingham techniques do not work, because the gap between frequencies goes to zero. The technique of biorthogonal sequence, that we will use, is more general. Examples of its application can be found in [3] for instance.

The idea is to find a sequence of functions $h_{n, k}$ with compact support such that $\widehat{h}_{n, k}\left(\nu_{n, k_{0}}\right)=\delta_{k_{0}}^{k}$, and whose $L^{2}$ norm is not too large. Indeed, will prove the following lemma: 
Lemma 3. For any odd integer $q$ and any positive real number $\varepsilon$, there exists a time $T_{2}(q, \varepsilon)$ smaller than $C_{q} \varepsilon^{(q+1) /(1-q)}$ such that for any $\left(n, k_{0}\right)$ in $\mathbb{N}^{*} \times\left(\mathbb{Z}^{*} \cup\{*, * *\}\right)$ there exists a function $h_{\varepsilon, q}^{k_{0}, n}$ that satisfies

i) $h_{\varepsilon, q}^{k_{0}, n}$ is supported by $\left[-T_{2}(q, \varepsilon), T_{2}(q, \varepsilon)\right]$.

ii) $\operatorname{For}\left(k_{0}, n\right) \in I,\left\|h_{\varepsilon, q}^{k_{0}, n}\right\|_{L^{2}}^{2} \leq C e^{2 \varepsilon|n|}$.

iii) If $k \neq \pm k_{0}$,

$$
\int h_{\varepsilon, q}^{k_{0}, n}(t) e^{i t \nu_{n, k}} d t=0
$$

iv) If $n \geq n_{1}(\varepsilon, q)$ and $\left(k_{0}, n\right) \in I$,

$$
\left|\int h_{\varepsilon, q}^{k_{0}, n}(t) e^{t \nu_{n, \pm k_{0}}} d t\right| \geq \frac{c}{n^{N_{q}}} .
$$

The constants depend only on $q$ and $\varepsilon$. Moreover the functions $h$ can be chosen as even or odd. We will denote them $h_{\varepsilon, q}^{k_{0}, n}$ or $h_{o \varepsilon, q}^{k_{0}, n}$.

Let us show at first how to prove Proposition 6 out of this lemma.

Let $n$ be an integer greater than $n_{1}(\varepsilon)$, and $\left(\Phi^{0}, \Phi^{1}, W^{0}, W^{1}\right)$ an initial condition in $\mathcal{Y}^{n}$. Let us denote $(\Psi, V)$ the solution of $(5)$ with these data.

We will denote $K$ the operator that maps $\left(\Phi^{0}, \Phi^{1}, W^{0}, W^{1}\right)$ in $\mathcal{Y}^{n}$ to $W_{t t}(y=0, \cdot)$. If we denote $a_{n, k}=\left\langle\left(\Phi^{0}, \Phi^{1}, W^{0}, W^{1}\right), \xi_{n, k} / \Xi_{n, k}\right\rangle$, we notice that

$$
W(y, t)=\sum_{k \in \mathbb{Z}^{*} \cup\{*, * *\}} a_{n, k} \frac{\xi_{n, k}^{3}}{\Xi_{n, k}} \cos (\pi n y) e^{i \nu_{n, k} t} .
$$

Thus

$$
K\left(\Phi^{0}, \Phi^{1}, W^{0}, W^{1}\right)(t)=-\sum_{k \in \mathbb{Z}^{*} \cup\{*, * *\}} a_{n, k} \frac{\xi_{n, k}^{3}}{\Xi_{n, k}} \nu_{n, k}^{2} e^{i \nu_{n, k} t} .
$$

Now for $\left(k_{0}, n\right)$ in $I$ and $L$ in $\mathbb{N}^{*}$, as $\widehat{h_{e}}$ is even,

$$
\begin{aligned}
\int h_{e_{\varepsilon, q}}^{k_{0}, n}(t) K( & \left.\sum_{\substack{|k|=* \\
|k| \leq L}} a_{n, k} \frac{\xi_{n, k}}{\Xi_{n, k}}\right)(t) d t \\
=- & \sum_{\substack{k=* \\
1 \leq k \leq L}}\left(a_{n, k}+a_{n,-k}\right) \frac{\xi_{n, k}^{3}}{\Xi_{n, k}} \nu_{n, k}^{2} \int h_{e_{\varepsilon, q}}^{k_{0}, n}(t) e^{i \nu_{n, k} t} d t .
\end{aligned}
$$


So, out of iii), if $L \geq k_{0}$,

$$
\begin{aligned}
\int h_{e \varepsilon, q}^{k_{0}, n}(t) K & \left(\sum_{\substack{|k|=* \\
|k| \leq L}} a_{n, k} \frac{\xi_{n, k}}{\Xi_{n, k}}\right)(t) d t \\
& =-\left(a_{n, k_{0}}+a_{n,-k_{0}}\right) \frac{\xi_{n, k_{0}}^{3}}{\Xi_{n, k_{0}}} \nu_{n, k_{0}}^{2} \int h_{e \varepsilon, q}^{k_{0}, n}(t) e^{i \nu_{n, k_{0}} t} d t
\end{aligned}
$$

So out of iv), we get that

$$
\begin{aligned}
\left|\int h_{e_{\varepsilon, q}}^{k_{0}, n}(t) K\left(\sum_{\substack{|k|=* \\
|k| \leq L}} a_{n, k} \frac{\xi_{n, k}}{\Xi_{n, k}}\right)(t) d t\right| \\
\quad \geq\left|a_{n, k_{0}}+a_{n,-k_{0}}\right|\left|\frac{\xi_{n, k_{0}}^{3}}{\Xi_{n, k_{0}}}\right|\left|\nu_{n, k_{0}}\right|^{2} \frac{c}{n^{N_{q}}} \\
\geq\left|a_{n, k_{0}}+a_{n,-k_{0}}\right| c e^{-n^{2 / 3}} .
\end{aligned}
$$

out of (63) and because, as we have already seen,

$$
\left|\xi_{n, k_{0}}^{3}\right|=\frac{\left|z_{n, k_{0}}\right|}{\left|\nu_{n, k_{0}}\right|}\left|\sin z_{n, k_{0}}\right| \geq \frac{C_{\gamma}}{n^{N}}
$$

If we take the limit with $L \longrightarrow+\infty$,

$$
\left|\int h_{e_{\varepsilon}, q}^{k_{0}, n}(t) K\left(\Phi^{0}, \Phi^{1}, W^{0}, W^{1}\right)(t) d t\right| \geq\left|a_{n, k_{0}}+a_{n,-k_{0}}\right| c e^{-n^{2 / 3}}
$$

We can show the same way that

$$
\left|\int h_{o \varepsilon, q}^{k_{0}, n}(t) K\left(\Phi^{0}, \Phi^{1}, W^{0}, W^{1}\right)(t) d t\right| \geq\left|a_{n, k_{0}}-a_{n,-k_{0}}\right| c e^{-n^{2 / 3}}
$$

So, by summing conveniently,

$$
\begin{aligned}
\left|a_{n, k_{0}}\right| \leq C e^{n^{2 / 3}} & \left(\left|\int h_{e \varepsilon, q}^{k_{0}, n}(t) K\left(\Phi^{0}, \Phi^{1}, W^{0}, W^{1}\right)(t) d t\right|\right. \\
& \left.+\left|\int h_{o \varepsilon, q}^{k_{0}, n}(t) K\left(\Phi^{0}, \Phi^{1}, W^{0}, W^{1}\right)(t) d t\right|\right) .
\end{aligned}
$$


So for any $n$ greater than $n_{1}(\varepsilon)$,

$$
\left\|\left(\Phi^{0}, \Phi^{1}, W^{0}, W^{1}\right)^{(1)}\right\|_{\mathcal{Y}}^{2}=\sum_{\substack{|k|=* \\|k| \leq|n|}}\left|a_{n, k}\right|^{2}
$$

So out of (70),

$$
\begin{aligned}
& \left\|\left(\Phi^{0}, \Phi^{1}, W^{0}, W^{1}\right)^{(1)}\right\|_{\mathcal{Y}}^{2} \\
& \leq C \sum_{\substack{|k|=* \\
|k| \leq|n|}} e^{n^{2 / 3}}\left|\int h_{e \varepsilon, q}^{k, n}(t) W_{t t}(0, t) d t\right|^{2}+\text { same with } h_{o} .
\end{aligned}
$$

Thus, out of i),

$$
\begin{aligned}
\left\|\left(\Phi^{0}, \Phi^{1}, W^{0}, W^{1}\right)^{(1)}\right\|_{\mathcal{Y}}^{2} \\
\leq C e^{n^{2 / 3}} \sum_{\substack{|k|=* \\
|k| \leq|n|}} \int\left|h_{\varepsilon, q}^{k, n}(t)\right|^{2} d t \int_{-T_{2}(q, \varepsilon)}^{T_{2}(q, \varepsilon)}\left|W_{t t}(0, t)\right|^{2} d t .
\end{aligned}
$$

Thus out of ii),

$$
\left\|\left(\Phi^{0}, \Phi^{1}, W^{0}, W^{1}\right)^{(1)}\right\|_{\mathcal{Y}}^{2} \leq C e^{C n^{2 / 3}} e^{2 \varepsilon|n|} \int_{-T_{2}(q, \varepsilon)}^{T_{2}(q, \varepsilon)}\left|W_{t t}(0, t)\right|^{2} d t .
$$

When $q$ goes to the infinity, if $(q+1) /(1-q)=-1-\delta, \delta$ goes to 0 . So we have proved Proposition 6.

We still have to prove Lemma 3.

First, we will introduce a sequence of functions $f^{k_{0}, n}$, that will satisfy conditions i), iii) and iv), but which $L^{2}$ norms will behave like $e^{n \pi}$, that is too large for ii). We will notice though that these norms will be mostly concentrated within $[-\pi n, \pi n]$, on the Fourier side.

Then we will build a sequence of functions $g$ of which we will know, by stationary phases computations, that their norms, on the Fourier side, are exponentially small over $[-\pi n, \pi n]$, and reasonably bounded outside.

We will then put $h=f * g$, and show that $h$ satisfy i) to iv), for suitable parameters. 


\subsection{Proof of Lemma 3.}

In order to prove this lemma, we will build two sequences of functions, denoted $f$ and $g$, and put $h=f * g$. The functions $f$ will have the right zeroes (on the Fourier side), but too large an $L^{2}$ norm. The functions $g$ will be small where $f$ is large, in order to get controlled $L^{2}$ norms. We will have to ensure also that they behave properly at the zeroes of $f$.

Namely, we will prove he following lemmas:

Lemma 4. For any $\left(n, k_{0}\right)$ in $\mathbb{N}^{*} \times\left(\mathbb{Z}^{*} \cup\{*, * *\}\right)$, there is an even $L^{2}$ function $f^{k_{0}, n}$ that satisfies:

i) $f^{k_{0}, n}$ is supported by $[-3 \pi, 3 \pi]$.

ii) For $z \in[-\pi n, \pi n],\left|\widehat{f}^{n, k_{0}}(z)\right| \leq C e^{3 n \sqrt{\pi^{2}-(z / n)^{2}}}$ and for $z \notin$ $[-\pi n, \pi n],\left|\hat{f}^{n, k_{0}}(z)\right| \leq P(n, k)$, where $P$ is a polynomial.

iii) If $k \neq \pm k_{0}, \widehat{f}^{n, k_{0}}\left(\nu_{n, k}\right)=0$.

iv) If $n \geq n_{1}(\varepsilon, q)$ and $\left(k_{0}, n\right) \in I=\{(k, n):|k|=*$ or $|k| \leq n\}$, $\left|\widehat{f}^{n, k_{0}}\left(\nu_{n, k_{0}}\right)\right| \geq c / n^{N_{q}}$.

Lemma 5. For large enough $T$, for any real number $\delta>1$, close to 1 , and any odd integer $q$, we can find three positive constants $C_{q}^{1}, C_{q, T}^{2}$, $c_{q, T, \delta}^{3}$ and two integers $r_{q}, n(q, \delta)$ such that for any integer $n$, there is a function $g_{T, q, \delta}^{n}$ in $L^{2}(\mathbb{R})$ such that:

i) $g_{T, q, \delta}^{n}$ is supported by $[-T, T]$.

ii) $\left|\widehat{g}_{T, q, \delta}^{n}\right|_{L^{\infty}} \leq 2 T$, and for any real number $\tau$ such that $|\tau| \leq n / \delta$,

$$
\left|\widehat{g}_{T, q, \delta}^{n}(\tau)\right| \leq C_{q, T}^{2} e^{-T n C_{q}^{1} \min \left\{(1 / \delta-\tau / n)^{q /(q-1)}, 1\right\}} .
$$

iii) For any integer $n$ greater than $n(q, \delta)$, if $k_{0}=*$ or $1 \leq k_{0} \leq n$, there is a time $T_{n, k_{0}}$ in $[T, T+1]$ such that

$$
\left|{\widehat{g_{+}}}_{T_{n, k_{0}}, q}^{n}\left(\frac{\left|\nu_{n, k_{0}}\right|}{\pi}\right)\right| \geq \frac{c_{q, T, \delta}^{3}}{\sqrt{n}} .
$$

The constants depend only on $q$ and $\varepsilon$. Moreover the functions $g$ can be chosen as even or odd. We will denote them $g_{e T, q, \delta}^{n}$ or $g_{o T, q, \delta}^{n}$. 
Let us prove Lemma 3 out of those two results.

Let $\varepsilon$ be a positive real number. Let us choose $\delta_{\varepsilon}$ such that

$$
3 \pi \sqrt{1-\left(\frac{1}{\delta_{\varepsilon}}\right)^{2}}=\frac{\varepsilon}{2}
$$

and $T^{\varepsilon}$ such that

$$
\sup _{\beta \in\left[0,1 / \delta_{\varepsilon}\right]}\left(3 \pi \sqrt{1-\beta^{2}}-C_{q}^{1} T^{\varepsilon}\left(\frac{1}{\delta_{\varepsilon}}-\beta\right)^{q /(q-1)}\right) \leq \varepsilon .
$$

The derivative is

$$
\frac{-3 \pi \beta}{\sqrt{1-\beta^{2}}}+\frac{q}{q-1} T^{\varepsilon} C_{q}^{1}\left(\frac{1}{\delta_{\varepsilon}}-\beta\right)^{1 /(q-1)},
$$

we choose $T^{\varepsilon}$ such that it is 0 for $\beta_{\varepsilon}$ such that $3 \pi \sqrt{1-\beta_{\varepsilon}}$.

We have

$$
\begin{aligned}
& \delta_{\varepsilon}=1+\frac{\varepsilon^{2}}{72 \pi^{2}}+o\left(\varepsilon^{2}\right), \\
& \beta_{\varepsilon}=1-\frac{\varepsilon^{2}}{18 \pi^{2}}+o\left(\varepsilon^{2}\right),
\end{aligned}
$$

so

$$
\frac{1}{\delta_{\varepsilon}}-\beta_{\varepsilon} \sim \frac{\varepsilon^{2}}{24 \pi^{2}}
$$

hence $T^{\varepsilon} \sim c_{q} \varepsilon^{(q+1) /(1-q)}$.

Let us define positive times $T_{n, k_{0}}^{\varepsilon}$ as follows. For integers $k_{0}$ such that $\left|k_{0}\right| \leq|n|$ or $\left|k_{0}\right|=*$, we take the time $T_{n, k_{0}}^{\varepsilon}$ given by Lemma 5 with $T=T^{\varepsilon}$; and for $\left|k_{0}\right|>|n|$, we put $T_{n, k_{0}}^{\varepsilon}=T^{\varepsilon}$.

$$
T_{n, k_{0}}^{\varepsilon} \in\left[T^{\varepsilon}, T^{\varepsilon}+1\right], \quad \text { so } c_{q}^{1} \varepsilon^{(q+1) /(1-q)} \leq T_{n, k_{0}}^{\varepsilon} \leq c_{q}^{2} \varepsilon^{(q+1) /(1-q)} \text {. }
$$

Let us denote

$$
\begin{aligned}
& {\widehat{h_{e}, q}}_{e_{0}, n}^{k_{0}}(\tau)=\widehat{f}^{k_{0}, n}(\tau) \widehat{g}_{e T_{n, k_{0}}^{\varepsilon}, q, \delta_{\varepsilon}}^{n}\left(\frac{\tau}{\pi}\right), \\
& {\widehat{h_{o \varepsilon, q}}}_{k_{0}, n}^{k^{\prime}}(\tau)=\widehat{f}^{k_{0}, n}(\tau) \widehat{g}_{o T_{n, k_{0}}^{\varepsilon}, q, \delta_{\varepsilon}}^{n}\left(\frac{\tau}{\pi}\right) .
\end{aligned}
$$


The subscript meaning that $h$ is even or odd. We will not write this subscript when not necessary.

We shall now prove step by step that $h_{\varepsilon, q}^{k_{0}, n}$ satisfies all the properties of Lemma 3.

Proof of i). By Lemma 4.i), the support of $f^{k_{0}, n}$ is located within $[-3 \pi, 3 \pi]$.

By Lemma 5.i), $g_{T_{n, k_{0}}^{\varepsilon}, q, \delta}^{n}$ is supported by $\left[-T_{n, k_{0}}^{\varepsilon}, T_{n, k_{0}}^{\varepsilon}\right]$.

As $h_{\varepsilon, q}^{k_{0}, n}$ is the convolution product of those two functions, it is supported by $\left[-T_{2}(q, \varepsilon), T_{2}(q, \varepsilon)\right]$ with $T_{2}(q, \varepsilon)=3 \pi+c_{q}^{2} \varepsilon^{(q+1) /(1-q)}$. The estimates on $T_{n, k_{0}}^{\varepsilon}$ insures that $T_{2}(q, \varepsilon) \leq C_{q} \varepsilon^{(q+1) /(1-q)}$.

Proof OF ii). We will use results about the small size of $\|g\|$ that will compensate $\|f\|$.

By Lemma 5.ii),

$$
|\widehat{g}|_{L^{\infty}} \leq 2 T_{2}(q, \varepsilon) .
$$

Furthermore, outside of $[-\pi n, \pi n]$, the $L^{2}$ norm of $f$ is bounded by a polynomial in $n$, so the problems are located within this interval.

We must estimate $\int_{-n}^{n}\left|\widehat{h}_{\varepsilon, q}^{k_{0}, n}(\tau)\right|^{2} d \tau$.

Now, out of Lemma 4.ii), we know that if $\tau / n$ belongs to $[-\pi, \pi]$, we have

$$
\left|f^{k_{0}, n}(\tau)\right|^{2} \leq C e^{6 n \sqrt{\pi^{2}-|\tau / n|^{2}}}=C e^{6 \pi n \sqrt{1-|\tau /(\pi n)|^{2}}} .
$$

Thus if $|\tau /(\pi n)| \geq 1 / \delta_{\varepsilon},\left|\widehat{h}_{\varepsilon, q}^{k_{0}, n}(\tau)\right|^{2} \leq C e^{\varepsilon n}$.

Moreover, out of Lemma 5.ii), if $|\tau /(\pi n)|$ is smaller than $1 / \delta_{\varepsilon}$, we have

$$
\left|\widehat{g}_{T_{n, k_{0}}^{\varepsilon}, q, \delta_{\varepsilon}}^{n}\left(\frac{\tau}{\pi}\right)\right|^{2} \leq C e^{-2 T_{n, k_{0}}^{\varepsilon} n C_{q}^{1}\left(1 / \delta_{\varepsilon}-|\tau /(\pi n)|\right)^{q /(q-1)}} .
$$

So out of (71), we get $\left|\widehat{h}_{\varepsilon, q}^{k_{0}, n}(\tau)\right|^{2} \leq C e^{2 \varepsilon n}$. Thus

$$
\left\|\widehat{h}_{\varepsilon, q}^{k_{0}, n}\right\|_{L^{2}}^{2} \leq C e^{2 \varepsilon n}
$$

Proof OF iii). This is a simple consequence of Lemma 4.iii). Indeed for any integer $k$ different from $k_{0}, \widehat{f}^{k_{0}, n}\left(\left|\nu_{n, k}\right|\right)=0$. So by definition of $h$, we also have $\widehat{h}_{\varepsilon, q}^{k_{0}, n}\left(\left|\nu_{n, k}\right|\right)=0$, which is exactly the Fourier transcription of iii). 
Proof OF iv). For any couple $\left(n, k_{0}\right)$ in $I$, out Lemma 4.iv) and Lemma 5.iii), we get

$$
\left|\widehat{h}_{\varepsilon, q}^{k_{0}, n}\left( \pm\left|\nu_{n, k_{0}}\right|\right)\right| \geq \frac{C}{n^{N}} \frac{c_{q, T_{\varepsilon}, \delta_{\varepsilon}}}{\sqrt{n}} \geq \frac{C_{q, \varepsilon}}{n^{N^{\prime}}}
$$

which is once again the Fourier transcription of the needed result.

Now we have to prove Lemmas 4 and 5 .

\subsubsection{Proof of Lemma 4: construction of $f$.}

Put

$$
\begin{gathered}
F^{n}(z)=\left(\left(z^{3}+z\left(n^{2} \pi^{2}-n^{4} \pi^{4}\right)\right) \tan z-z^{2}-n^{2} \pi^{2}\right) \cos z \\
G^{n}(z)=\sqrt{z^{2}-n^{2} \pi^{2}}
\end{gathered}
$$

and

$$
f^{n}(z)=F^{n}\left(G^{n}(z)\right) .
$$

The following properties hold for these functions:

f-i) $f_{0}^{n} \in \mathcal{O}(\mathbb{C})$.

f-iii) For any $k$ in $\mathbb{Z}^{*} \cup\{*, * *\}, f_{0}^{n}\left(\nu_{n, k}\right)=\left(z_{n, k}^{3} \tan z_{n, k}-z_{n, k}^{2}-\right.$ $\left.n^{2} \pi^{2}\right) \cos z_{n, k}=0$ out of (57).

Let us evaluate $f^{n^{\prime}}\left(\nu_{n, k}\right)$.

$$
f^{n \prime}\left(\nu_{n, k}\right)=G^{n \prime}\left(\nu_{n, k}\right) F^{n \prime}(\underbrace{G^{n}\left(\nu_{n, k}\right)}_{z_{n, k}}) .
$$

Now $\left|G^{n^{\prime}}\left(\nu_{n, k}\right)\right|=\left|\nu_{n, k} / z_{n, k}\right| \geq 1$. So to bound $\left|f^{n^{\prime}}\left(\nu_{n, k}\right)\right|$ from bellow, we only have to bound $\left|F^{n^{\prime}}\left(z_{n, k}\right)\right|$ from bellow. To simplify the notation, put $\alpha_{n}=n^{4} \pi^{4}-n^{2} \pi^{2}$.

$$
\begin{aligned}
& F^{n^{\prime}}\left(z_{n, k}\right) \\
& =\cos z_{n, k} \\
& \cdot(\underbrace{-2 z_{n, k}+\left(z_{n, k}^{3}-\alpha_{n} z_{n, k}\right)\left(1+\tan ^{2} z_{n, k}\right)+\left(3 z_{n, k}^{2}-\alpha_{n}\right) \tan z_{n, k}}_{h\left(z_{n, k}\right)})
\end{aligned}
$$


(see pictures 1 and 2.)

We recall that the first value of $k$ for which $z_{n, k}$ is larger than $\sqrt{\alpha_{n}}$ is denoted by $k_{0}$. If $k \neq k_{0}$ and $k \neq k_{0}-1,\left|z_{n, k}-\sqrt{\alpha_{n}}\right| \geq \pi / 2$. As we also have $z_{n, k} \geq \pi / 2$ and $z_{n, k}$ is a root of $\tan z_{n, k}=\left(z^{2}+n^{2} \pi^{2}\right) /\left(z^{3}-\alpha_{n} z\right)$, we get $\left|\cos z_{n, k}\right| \geq 1 / P(n, k)$ where $P$ is a polynomial.

Let us consider $h$. For any positive $\varepsilon$ and $z=z_{n, k} \geq \sqrt{\alpha_{n}}+\varepsilon$,

$$
\begin{aligned}
h(z) & =-2 z+\left(z^{3}-\alpha_{n} z\right)\left(1+\tan ^{2} z\right)+\left(3 z^{2}-\alpha_{n}\right) \tan z \\
& \geq-2 z+\left(z-\sqrt{\alpha_{n}}\right)\left(z+\sqrt{\alpha_{n}}\right) z \\
& \geq\left(2 \varepsilon \alpha_{n}-2\right) z \\
& >1 \quad \text { for large } n .
\end{aligned}
$$

For $\sqrt{\alpha_{n} / 3} \leq z=z_{n, k}<\sqrt{\alpha_{n}}, h(z) \leq-2 z$ so $|h(z)| \geq 1$.

And for $z=z_{n, k} \leq \sqrt{\alpha_{n} / 3}$,

$$
h(z) \leq-2 z-\left(\left|z^{3}-\alpha_{n} z\right|\left|1+\tan ^{2} z\right|-\left|3 z^{2}-\alpha_{n}\right||\tan z|\right),
$$

now $\left|1+\tan ^{2} z\right|>|\tan z|$ and as $\pi / 2 \leq z \leq \sqrt{\alpha_{n} / 3},\left|z^{3}-\alpha_{n} z\right| \geq$ $\left|3 z^{2}-\alpha_{n}\right|$.

So $\left|h\left(z_{n, k}\right)\right| \geq\left|2 z_{n, k}\right| \geq 1$.

Hence we know that if $k \neq k_{0}$ and $k \neq k_{0}-1$,

$$
\left|F^{n^{\prime}}\left(z_{n, k}\right)\right| \geq \frac{1}{P(n, k)}
$$

Now if $k=k_{0}$ or $k=k_{0}-1, z_{n, k} \in\left[\sqrt{\alpha_{n}}-\pi / 2, \sqrt{\alpha_{n}}+\pi / 2\right]$ so for large $n, z_{n, k} \sim \sqrt{\alpha_{n}}$. Now

$$
z_{n, k}-\sqrt{\alpha_{n}}=\underbrace{\frac{z_{n, k}^{2}+n^{2} \pi^{2}}{z\left(z+\sqrt{\alpha_{n}}\right)}}_{\sim 1 / 2} \frac{\cos z_{n, k}}{\sin z_{n, k}} .
$$

So for a small fixed $\eta$, either $\left|\cos z_{n, k}\right| \geq \eta$, then

$$
\left|z_{n, k}-\sqrt{\alpha_{n}}\right| \geq \varepsilon(\eta)
$$

and in that case we know that $\left|h\left(z_{n, k}\right)\right| \geq 1$, hence $\left|F^{n^{\prime}}\left(z_{n, k}\right)\right| \geq \eta$. 
Either $\left|\cos z_{n, k}\right|<\eta$, now

$$
\begin{aligned}
F^{n \prime}\left(z_{n, k}\right)= & \underbrace{-2 z_{n, k} \cos z_{n, k}}_{|\cdot| \leq 2(\eta+\varepsilon) \sqrt{\alpha_{n}}}+\underbrace{\left(3 z_{n, k}^{2}-\alpha_{n}\right) \sin z_{n, k}}_{|\cdot| \geq 2 \alpha_{n} \sqrt{1-\eta^{2}}} \\
& +\underbrace{\frac{z_{n, k}-\sqrt{\alpha_{n}}}{\cos z_{n, k}} z_{n, k}\left(z_{n, k}+\sqrt{\alpha_{n}}\right)}_{|\cdot| \leq(1 / 2+\varepsilon)\left(2 / \sqrt{1-\eta^{2}}\right) \alpha_{n}} .
\end{aligned}
$$

Now for small $\eta$,

$$
2 \sqrt{1-\eta^{2}}>\frac{2}{\sqrt{1-\eta^{2}}}\left(\frac{1}{2}+\varepsilon\right)
$$

so

$$
\left|F^{n \prime}\left(z_{n, k}\right)\right| \geq c \alpha_{n} \geq 1 .
$$

So we have proved that for any $n, k$,

$$
\left|f^{n \prime}\left(\nu_{n, k}\right)\right| \geq \frac{1}{P(n, k)}
$$

Let us put for any $k$ in $\mathbb{Z}^{*} \cup\{*, * *\}$,

$$
\widehat{f}^{k, n}(z)=f_{0}^{n}(z) \frac{1}{z^{2}-\left|\nu_{n, k}\right|^{2}}\left(\frac{\sin \sqrt{z^{2}-\pi^{2} n^{2}}}{\sqrt{z^{2}-\pi^{2} n^{2}}}\right)^{2}
$$

(the last term ensures that $f$ remains in $L^{2}$ ).

Let us show that these functions satisfy the properties of Lemma 4: by construction, they are even.

As $f_{0}$ has got zeroes at $\pm\left|\nu_{n, k}\right|, \hat{f}^{k, n} \in \mathcal{O}(\mathbb{C})$. Moreover $\widehat{f}^{k, n} \in$ $L^{2}(\mathbb{R})$ and for any complex number $z,\left|\widehat{f}_{0}^{k, n}(z)\right| \leq C e^{3|\operatorname{Im} z|}$.

So by the Paley Wienner theorem, we have property i).

Property ii) is straightforward, due to the explicit value of $\widehat{f}^{n, k}$.

As by f-iii), $\nu_{n, k}$ is a zero of $f_{0}^{n}$ for any $k$, it is by definition a zero of $\widehat{f}^{n, k_{0}}$ if $k \neq k_{0}$, so iii) holds

Furthermore,

$$
f_{0}^{k, n}\left( \pm\left|\nu_{n, k}\right|\right)=f_{0}^{n \prime}\left( \pm\left|\nu_{n, k}\right|\right)\left(\frac{\sin z_{n, k}}{z_{n, k}}\right)^{2} \frac{1}{\mp 2\left|\nu_{n, k}\right|}
$$


thus

$$
\left|f_{0}^{k, n}\left( \pm\left|\nu_{n, k}\right|\right)\right| \geq \frac{C}{\left(1+n^{2}\right)|k|^{3}}\left|\sin z_{n, k}\right|^{2} \geq \frac{C}{\left(1+n^{2}+k^{2}\right)^{N_{2}}} .
$$

So iv) holds.

\subsubsection{Proof of Lemma 5: construction of functions $g$.}

Let $q$ be an odd integer and let us denote $h_{q}(x)$ the solution of $y^{\prime}=1+y^{q-1}$ that satisfies $y(0)=0$. This function is defined over $\left(-x_{q}, x_{q}\right)$ for a positive $x_{q}$. It is odd, strictly increasing and analytic. Moreover, we have $h_{q}(x)=x+\alpha_{q} x^{q}+o\left(x^{q}\right)$ when $x$ is near 0 , with a positive $\alpha_{q}$ and when $x$ goes to $x_{q}, h_{q}$ goes to the infinity.

We shall denote $H_{q}$ the reciprocal function to $h_{q}$. It is defined over $\mathbb{R}$, odd, strictly increasing, bounded by $x_{q}$. We have $H_{q}(x)=$ $x-\alpha_{q} x^{q}+o\left(x^{q}\right)$ if $x$ is close to 0 .

Let $\delta$ be a real number, greater than 1 , and close to 1 , that will be fixed later.

Let us define functions $g$ as follows

$$
\begin{aligned}
& g_{+T, q}^{n}(t)=\mathbf{1}_{(-T, T)} e^{i n\left(T / \delta x_{q}\right) h_{q}\left(\left(x_{q} / T\right) t\right)}, \\
& {\widehat{g_{+}, q}}_{n}^{n}(\tau)=\int_{-T}^{T} e^{i n\left(T / \delta x_{q}\right) h_{q}\left(\left(x_{q} / T\right) t\right)-i \tau t} d t .
\end{aligned}
$$

Let us write $\Psi_{q}(s)=\left(T / x_{q}\right) H_{q}\left(\left(\delta x_{q} / T\right) s\right)$,

$$
{\widehat{g_{+}, q}}_{T}^{n}(\tau)=\int_{-\infty}^{+\infty} e^{i n s-i \tau \Psi_{q}(s)} \Psi_{q}^{\prime}(s) d s .
$$

If we denote

$$
\theta_{q}(s)=\frac{1}{x_{q}} H_{q}\left(\delta x_{q} s\right)
$$

we have

$$
\begin{aligned}
\widehat{g_{+T, q}}(\tau) & =\int_{-\infty}^{+\infty} \theta_{q}^{\prime}\left(\frac{s}{T}\right) e^{i n T\left(s / T-(\tau / n) \theta_{q}(s / T)\right)} d s \\
& =T \int_{-\infty}^{+\infty} \theta_{q}^{\prime}(v) e^{i n T\left(v-(\tau / n) \theta_{q}(v)\right)} d v
\end{aligned}
$$


Let us put $\alpha=n T$ and $\beta=\tau / n$. We will estimate

$$
\psi(\alpha, \beta)=\int \theta_{\delta}^{\prime}(v) e^{i \alpha\left(v-\beta \theta_{\delta}(v)\right)} d v
$$

for $\alpha$ going to the infinity.

There will be two kinds of estimates depending upon the value of $\beta$ as compared to $1 / \delta$.

- If $\beta<1 / \delta$. In this zone, the phases is non-stationary. So we will get and exponential decrease.

Let us shift slightly in the imaginary direction. For any real number $v$, any $\beta$ smaller than $1 / \delta$ and any little $\varepsilon$, we get

$$
\begin{aligned}
\operatorname{Im}\left(v+i \varepsilon-\beta \theta_{q}(v\right. & +i \varepsilon)) \\
& =\varepsilon-\beta \operatorname{Im} \theta_{q}(v+i \varepsilon) \\
& =\varepsilon-\beta \operatorname{Im}\left(\theta_{q}(v+i \varepsilon)-\theta_{q}(v)\right) \\
& =\varepsilon-\beta \operatorname{Im} \int_{v}^{v+i \varepsilon} \theta_{q}^{\prime}(z) d z \\
& =\varepsilon-\beta \operatorname{Im} \int_{v}^{v+i \varepsilon} \frac{\delta d z}{1+\delta^{q-1} x_{q}^{q-1} z^{q-1}} \\
& =\varepsilon-\beta \varepsilon \delta \operatorname{Re} \int_{0}^{1} \frac{d u}{1+\delta^{q-1} x_{q}^{q-1}(v+i \varepsilon u)^{q-1}} \\
& \geq \varepsilon \quad \text { if } \beta \leq 0 .
\end{aligned}
$$

If $\beta$ is positive,

$\operatorname{Im}\left(v+i \varepsilon-\beta \theta_{q}(v+i \varepsilon)\right) \geq \varepsilon-\beta \varepsilon \delta\left|\int_{0}^{1} \frac{d u}{1+\delta^{q-1} x_{q}^{q-1}(v+i \varepsilon u)^{q-1}}\right|$.

Now for any real $v$,

$$
\underbrace{\left|\int_{0}^{1} \frac{d u}{1+\delta^{q-1} x_{q}^{q-1}(v+i \varepsilon u)^{q-1}}\right|}_{I} \leq \frac{1}{1-c_{q} \varepsilon^{q-1}}
$$

because either $v \gg \varepsilon$ and then

$$
I \leq \frac{c}{1+v^{q-1}} \leq 1
$$


or $v \leq M_{q} \varepsilon$ and then

$$
|v+i \varepsilon u|^{q-1} \leq C_{q} \varepsilon^{q-1}
$$

implies

$$
\left|1+\delta^{q-1} x_{q}^{q-1}(v+i \varepsilon u)^{q-1}\right| \geq 1-c_{q} \varepsilon^{q-1}
$$

implies

$$
I \leq \frac{1}{1-c_{q} \varepsilon^{q-1}}
$$

Thus

$$
\begin{aligned}
\operatorname{Im}\left(v+i \varepsilon-\beta \theta_{q}(v+i \varepsilon)\right) & \geq \varepsilon-\frac{\beta \delta \varepsilon}{1-c_{q} \varepsilon^{q-1}} \\
& \geq \varepsilon(1-\delta \beta)-c_{q}^{\prime} \beta \varepsilon^{q} \\
& \geq \varepsilon\left(\frac{1}{\delta}-\beta\right)-c_{q}^{\prime} \beta \varepsilon^{q} .
\end{aligned}
$$

Now

$$
\max _{\varepsilon} \varepsilon\left(\frac{1}{\delta}-\beta\right)-c_{q} \beta \varepsilon^{q}=c_{q}^{\prime}\left(\frac{1}{\delta}-\beta\right)^{q /(q-1)} \beta^{1 /(1-q)} \geq c_{q}^{\prime \prime}\left(\frac{1}{\delta}-\beta\right)^{q /(q-1)} .
$$

We can choose a real number $\varepsilon$ and a very small $c_{q}$ such that for any real number $v$,

$$
\begin{cases}\operatorname{Im}\left(v+i \varepsilon-\beta \theta_{q}(v+i \varepsilon)\right) \geq c_{q}\left(\frac{1}{\delta}-\beta\right)^{q /(q-1)}, & \text { if } \beta \in\left(0, \frac{1}{\delta}\right], \\ \operatorname{Im}\left(v+i \varepsilon-\beta \theta_{q}(v+i \varepsilon)\right) \geq c_{q}, & \text { if } \beta \leq 0 .\end{cases}
$$

Now we can shift the integration line over $v$ from $\mathbb{R}$ to $\mathbb{R}+i \varepsilon$

$$
\psi(\alpha, \beta)=\int \theta_{q}^{\prime}(v+i \varepsilon) e^{i \alpha\left(v+i \varepsilon-\beta \theta_{q}(v+i \varepsilon)\right)} d v .
$$

To end with, as

$$
\theta_{q}^{\prime}(v+i \varepsilon)=\frac{\delta}{1+\left(\delta x_{q}(v+i \varepsilon)\right)^{q}-1}
$$

we get

$$
\left|\theta_{q}^{\prime}(v+i \varepsilon)\right| \leq \frac{C_{q}}{1+v^{q-1}},
$$


hence for any real number $\alpha$ and any $\beta \leq 1 / \delta$,

$$
\begin{aligned}
|\psi(\alpha, \beta)| & \leq \int \frac{C_{q}}{1+v^{q-1}} e^{-\alpha c_{q} \min \left\{(1 / \delta-\beta)^{q /(q-1)}, 1\right\}} d v \\
& \leq C_{q} e^{-\alpha c_{q} \min \left\{(1 / \delta-\beta)^{q /(q-1)}, 1\right\}} .
\end{aligned}
$$

So if $\tau / n \leq 1 / \delta$,

$$
\left|\widehat{g+T, q}^{n}(\tau)\right| \leq C_{q} T e^{-n T c_{q} \min \left\{(1 / \delta-\tau / n)^{q /(q-1)}, 1\right\}} .
$$

- If $\beta \geq 1(>1 / \delta)$. Through the stationary phase formula (see [7, p. 431]), we get

$\psi(\alpha, \beta)=C\left(\left|H_{\beta, \delta}\right| \cos \alpha p_{0}(\beta, \delta)\right)\left(\frac{\theta_{q}^{\prime}\left(v_{0}(\beta, \delta)\right)}{\sqrt{\alpha}}+\sum_{j=1}^{N} \frac{a_{j}(\beta, \delta)}{\alpha^{j} \sqrt{\alpha}}\right)+r_{\beta, \delta}(\alpha)$,

where $r_{\beta, \delta}(\alpha) \leq C_{\beta} / \alpha^{N+1}$ and $\alpha \geq A_{\beta, \delta} ; H_{\beta, \delta}$ denoting the square root of the Hessian at the critical points.

Moreover, in this formula, $C$ and $A$ are continuous with respect to $\beta$ and $\delta$, and $a_{j}(\beta, \delta)$ depends on the first $2 j+1$ derivatives of $v \longmapsto \theta_{q}(v)$ at $v=v_{0}(\beta, \delta)$.

Let us compute $p_{0}(\beta, \delta)$.

$$
\begin{array}{rlr}
\frac{\partial}{\partial v}\left(v-\beta \theta_{q}(v)\right)=0 & \text { if an only if } 1-\frac{\beta \delta}{1+\delta^{q-1} x_{q}^{q-1} v^{q-1}}=0 \\
& \text { implies } \quad 1+\delta^{q-1} x_{q}^{q-1} v_{0}^{q-1}(\beta, \delta)=\beta \delta \\
& \text { implies } & v_{0}(\beta, \delta)=\frac{1}{\delta x_{q}}(\delta \beta-1)^{1 /(q-1)} .
\end{array}
$$

If $\beta$ takes the values $\left|\nu_{n, k}\right| /(n \pi)$ for any couple $(n, k)$ such that $|k| \leq n$, we have $1 \leq \beta \leq \pi \sqrt{2}$.

Moreover, if $\beta=\left|\nu_{n, *}\right| /(n \pi)$, by (62),

$$
\beta \geq 1-\frac{C}{\sqrt{n}} \geq \frac{1}{2}\left(1+\frac{1}{\delta}\right)
$$

as soon as $n \geq n_{0}(\delta)$.

So for any $n$ greater than $n_{0}(\delta)$, if $(n, k)$ belongs to $I$ and $\beta=$ $\left|\nu_{n, k}\right| /(n \pi), C \geq v_{0}(\beta, \delta),\left|p_{0}(\beta, \delta)\right|,\left|H_{\beta, \delta}\right| \geq c_{\delta}$, thus $1 \geq \theta_{q}^{\prime}\left(v_{0}(\beta, \delta)\right)$ $\geq c_{q}$. Moreover $a_{j}(\beta, \delta) \leq C_{j, \delta}$. 
Let $T$ be a positive real time. As $\left|p_{0}(\beta, \delta)\right| \geq c_{\delta}$, for any $n$ greater than $n_{0}(\delta)$, and $k_{0}$ such that $\left(n, k_{0}\right)$ belongs to $I$, one can pick a time $T_{n, k_{0}}$ in $[T, T+1]$ such that

$$
\cos \left(n T_{n, k_{0}} p_{0}\left(\frac{\left|\nu_{n, k_{0}}\right|}{n \pi}, \delta\right)\right) \geq c_{\delta}^{\prime} .
$$

Thus for $T>T_{u}, n \geq n(q, \delta), \alpha=T n,\left(k_{0}, n\right) \in I$ and $\beta=\left|\nu_{n, k_{0}}\right| /(n \pi)$,

$$
\begin{gathered}
\left|\frac{\theta_{q}^{\prime}\left(v_{0}(\beta, \delta)\right)}{\sqrt{\alpha}}+\sum_{j=1}^{N} \frac{a_{j}(\beta, \delta)}{\alpha^{j} \sqrt{\alpha}}\right| \geq \frac{\left|\theta_{q}^{\prime}\left(v_{0}(\beta, \delta)\right)\right|}{2 \sqrt{\alpha}} \\
\left|r_{\beta, \delta}(\alpha)\right| \leq c_{\delta}^{\prime} \frac{\left|H_{\beta, \delta}\right| \theta_{q}^{\prime}\left(v_{0}(\beta, \delta)\right)}{4 \sqrt{\alpha}}
\end{gathered}
$$

And in the same conditions, there is a time $T_{n, k_{0}}$ in $[T, T+1]$ such that

$$
\left|\psi\left(n T_{n, k_{0}}, \frac{\left|\nu_{n, k_{0}}\right|}{n \pi}\right)\right| \geq \frac{c_{\delta}^{\prime}|H| \theta_{q}^{\prime}\left(v_{0}\left(\frac{\nu_{n, k_{0}}}{n \pi}, \delta\right)\right)}{4 \sqrt{n} \sqrt{T_{n, k_{0}}}} \geq \frac{c}{\sqrt{n}} .
$$

We have proved that for any time $T$ greater than $T_{u}$, for any $n$ larger than $n(q, \delta)$ and $k_{0}$ such that $\left|k_{0}\right|=*$ or $\left|k_{0}\right| \leq n$, there is a time $T_{n, k_{0}}$ in $[T, T+1]$ such that

$$
\left|\widehat{g}_{+T_{n, k_{0}}, q}^{n}\left(\frac{\left|\nu_{n, k_{0}}\right|}{\pi}\right)\right| \geq \frac{C_{T, q, \delta}}{\sqrt{n}} .
$$

By changing $t$ into $-t$, we can prove two estimates similar to (74) and (75) for the functions

$$
g_{-T, q, \delta}^{n}(t)=\mathbf{1}_{(-T, T)} e^{i n\left(T / \delta x_{q}\right) h_{q}\left(\left(x_{q} / T\right) t\right)} .
$$

As $g_{-T, q, \delta}^{n}=\overline{g_{+T, q, \delta}^{n}}$, we have: $T_{n, k_{0},+}=T_{n, k_{0},-}$.

So if we put

$$
g_{e T, q, \delta}^{n}(t)=\mathbf{1}_{(-T, T)} \cos \left(n \frac{T}{\delta x_{q}} h_{q}\left(\frac{x_{q}}{T} t\right)\right),
$$

we have

$$
g_{e T, q, \delta}^{\stackrel{n}{n}}(t)=\operatorname{Re} g_{+T, q, \delta}^{\stackrel{n}{n}}(t)=\frac{1}{2}\left(g_{+T, q, \delta}^{\stackrel{n}{n}}(t)+g_{-T, q, \delta}^{\stackrel{n}{n}}(t)\right) .
$$


Let us show that this even function satisfies the properties of Lemma 5 .

i) By definition, it is supported by $[-T, T]$.

ii) is an easy consequence of the definition and (73) for the $L^{\infty}$ estimate, and (74) for the other one.

iii) If $n \geq n(q, \delta)$ and $\left(\left|k_{0}\right| \leq n\right.$ or $\left.\left|k_{0}\right|=*\right), C_{q, T}^{2} e^{-n T C_{q}^{1}} \leq$ $c_{q, T, \delta}^{3} /(2 \sqrt{n})$, so , if $n$ is large enough, by (74) and (75),

$$
\left|{\widehat{g_{ \pm}}}_{T_{n, k_{0}}, q, \delta}^{n}(\tau)\right| \leq\left|\widehat{g}_{\mp T_{n, k_{0}}, q, \delta}^{n}(\tau)\right|, \quad \text { for } \tau=\mp \frac{\left|\nu_{n, k_{0}}\right|}{\pi}
$$

As we can increase the constants to cope with the finite number of $(n, k)$ in $I$ for which $n$ is not large enough, we get for $\left(n, k_{0}\right)$ in $I$ and $\tau= \pm\left|\nu_{n, k_{0}}\right| / \pi$

$$
\left|\widehat{g}_{e T_{n, k_{0}}, q, \delta}^{n}(\tau)\right| \geq \frac{c_{q}, T, \delta}{\sqrt{n}} .
$$

Of course, similar results hold for the odd function

$$
g_{o T, q, \delta}^{n}(t)=\mathbf{1}_{(-T, T)} \sin \left(n \frac{T}{\delta x_{q}} h_{q}\left(\frac{x_{q}}{T} t\right)\right) .
$$

This ends the proof of Lemma 5.

\section{References.}

[1] Allibert, B., Contrôle analytique de l'équation des ondes et de l'équation de Schrodinger sur des surfaces de revolution. Comm. Partial Diff. Equations 23 (1998), 1493-1556.

[2] Avalos, G., Lasiecka, I., A differential Riccati equation for the active control of a problem in structural acoustics. J. Optim. Theory Appl. 91 (1996), 695-728.

[3] Avdonin, S. A., Ivanov, S. A., Families of exponentials. The method of moments in controllability problems for distributed parameter systems. Cambridge Univ. Press, 1995.

[4] Banks, H. T., Fang, W., Silcox, R. J., Smith, R. C., Approximation Methods for Control of Acustic/Structure Models with Piezoceramic Actuators. Journal of Intelligent Material Systems and Structures. 4 (1993), 98-116. 
[5] Ingham, A. E., Some trigonometrical inequalities with applications to the theory of series. Math. Z. 41 (1936), 367-369.

[6] Lions, J. L., Contrôlabilité exacte, perturbations et stabilization de systèmes distribués. Tome 1. Contrôlabilité exacte. Masson, 1988.

[7] Micu, S., Zuazua, E., Boundary controllability of a linear hybrid system arising in the control of noise. SIAM J. Control Optim. 35 (1997), 1614-1637.

[8] Trèves, F., Introduction to pseudodifferential and Fourier integral operators. Plenum Press, The university series in mathematics. 2 vol., 1980.

[9] Tucsnak, M., Regularity and Exact Controllability for a Beam with Piezoelectric Actuators. SIAM J. Control Optim. 34 (1996), 922-930.

Recibido: 31 de marzo de 1.998

Brice Allibert

Centre de Mathématiques

Ecole Polytechnique

URM 7640 du CNRS

F-91128 Palaiseau, FRANCE

allibert@math.polytechnique.fr

and

Sorin Micu*

Departamento de Matemática Aplicada

Facultad de Ciencias Matemáticas

Universidad Complutense

28040 Madrid, SPAIN

and

Facultatea de Matematica-Informatica

Universitatea din Craiova

1100, ROMANIA

sorin@sunma4.mat.ucm.es

\footnotetext{
* Partially Supported by Grant PB96-0663 of DGES (Spain) and Grant 195/1997 of CNCSU (Romania).
} 